\title{
Aerodynamic comparison of a butterfly-like flapping wing-body model and a revolving-wing model
}

\author{
Kosuke Suzuki ${ }^{1} \ddagger$ and Masato Yoshino ${ }^{1,2}$ \\ ${ }^{1}$ Institute of Engineering, Academic Assembly, Shinshu University, Nagano 380-8553, \\ JAPAN \\ ${ }^{2}$ Institute of Carbon Science and Technology, Interdisciplinary Cluster for Cutting \\ Edge Research, Shinshu University, Nagano 380-8553, JAPAN \\ E-mail: kosuzuki@shinshu-u.ac.jp
}

\begin{abstract}
The aerodynamic performance of flapping- and revolving-wing models is investigated by numerical simulations based on an immersed boundary-lattice Boltzmann method. As wing models, we use (i) a butterfly-like model with a body and flapping-rectangular wings and (ii) a revolving-wing model with the same wings as the flapping case. Firstly, we calculate aerodynamic performance factors such as the lift force, the power, and the power loading of the two models for Reynolds numbers in the range of 50-1000. For the flapping-wing model, the power loading is maximal for the maximum angle of attack of $90^{\circ}$, a flapping amplitude of roughly $45^{\circ}$, and a phase shift between the flapping angle and the angle of attack of roughly $90^{\circ}$. For the revolving-wing model, the power loading peaks for an angle of attack of roughly $45^{\circ}$. In addition, we examine the ground effect on the aerodynamic performance of the revolving-wing model. Secondly, we compare the aerodynamic performance of the flapping- and revolving-wing models at their respective maximal power loadings. It is found that the revolving-wing model is more efficient than the flapping-wing model both when the body of the latter is fixed and where it can move freely. Finally, we discuss the relative agilities of the flapping- and revolving-wing models.
\end{abstract}

Keywords: Flapping wing, Revolving wing, Aerodynamic performance, Lattice Boltzmann method, Immersed boundary method

‡ Corresponding author: kosuzuki@shinshu-u.ac.jp 


\section{Introduction}

The flapping flight of insects propelled by flapping is an interesting phenomenon not only in biology but also in aerodynamics. In addition, its practical use for the development of micro air vehicles (MAVs) has recently attracted a lot of attention (e.g., Ma et al. 2013, Ristroph \& Childress 2014). In order to reveal the various mechanisms that generate aerodynamic forces in flapping flight, much effort has been made over the past 50 years with analytical, experimental, and computational approaches. Several wing models that mimic actual insects (known as flapping-wing models) have been used to investigate the force-generation mechanisms (e.g., Ellington et al. 1996, Liu et al. 1998, Dickinson et al. 1999). Alternatively, models in which fixed wings revolve like propellers at constant angle of attack and angular velocity (known as revolving-wing models) have been used as simpler substitutes for flapping-wing models. Usherwood \& Ellington (2002a) and Usherwood \& Ellington (2002b) performed experiments using a revolving-wing model of a hawkmoth's wing to study the properties of the leadingedge vortex (LEV) on the wing. They found that large lift and drag coefficients arise from the presence of the LEV during steady revolution. Lentink \& Dickinson (2009a) and Lentink \& Dickinson (2009b) conducted analytical and experimental investigation into the stability of the LEV of a fly's wing using revolving- and flapping-wing models with three non-dimensional parameters: the Rossby number, the non-dimensional stroke amplitude, and the Reynolds number Re. They found that the stability of the LEV depends on the Rossby number rather than the non-dimensional stroke amplitude. In other words, the LEV is stabilized by the centripetal and Coriolis accelerations, whereas the mechanism for LEV stability is independent of the Reynolds number. Ozen \& Rockwell (2012) obtained experimental images of the vorticity, velocity contours, and streamline topology around a revolving-wing model with a rectangular plate in order to characterize the LEV in relation to the overall flow structure. They found that a stable LEV is maintained over wide range of angle of attack during steady revolution, with a structure that is relatively insensitive to the Reynolds number. Harbig et al. (2013a) and Harbig et al. (2013b) performed numerical investigations of the effects of the Reynolds number, aspect ratio, and wing camber on the LEV structure and aerodynamic forces in a revolving-wing model of insect-like wings. They found that a dual LEV structure develops at the Reynolds numbers associated with insects, with a structure that is independent of aspect ratio. In addition, they found that positively cambered wings produce higher lift-to-drag ratios than those of flat or negatively cambered wings. Garmann et al. (2013) and Garmann \& Visbal (2014) simulated the flow around a rectangular-plate revolving-wing model in quiescent fluid to examine the vortex structure around and aerodynamic loading on the wing. They found a coherent vortex system along the LEV throughout the wing motion, with the average and instantaneous wing loadings increasing with the Reynolds number. Noda et al. (2014) investigated numerically the aerodynamics of a flexible wing at low Reynolds numbers using a hawkmoth revolving-wing model. They found that wing deformation 
delays vortex shedding at the leading edge, thereby improving the aerodynamic forces during steady revolution.

As discussed above, revolving-wing models have been used as a simpler approach to investigating the flow structures and aerodynamic forces associated with flapping-wings, especially the properties of the LEV. However, although such models have led a better understanding of the force-generation mechanisms in flapping flight, the aforementioned revolving-wing studies did not discuss important aerodynamic performance indices such as the aerodynamic power expenditure or the power loading (lift force per unit power). Moreover, at the Reynolds numbers associated with insects, the optimal angle of attack (i.e., the one that gives the maximum power loading) has not been clarified even for the simplest revolving-wing model with rectangular wings. In addition, as an interesting issue concerning the efficiency of revolving wings, the following simple questions have not been answered sufficiently. Firstly, for flapping- and revolving-wing models with the same wings, which model generates the larger lift force and expends the least aerodynamic power? Secondly, what are the advantages and disadvantages of flapping versus revolving wings? The above two questions are important in the development of flapping-wing MAVs. Because many practical MAVs use revolving wings, they are the main competition for flapping wings. Hence, comparing the two wing models would give valuable data for improving the aerodynamic performance of MAVs. Motivated by such questions, several studies have compared revolving wings to flapping ones. Mayo \& Leishman (2010) conducted a comparative study using published data for several types of hover-capable MAVs such as revolving wings, cyclorotors, flotors, and flapping wings and for avian/entomological flyers. They suggested that power loading is the most objective index for comparing the aerodynamic efficiency among different types of aircraft. They concluded that revolving-wing flight efficiency is either comparable to or better than avian/entomological flight efficiency, including biomimetic flapping wings. Zheng et al. (2013) used direct numerical simulations to compare the hovering performance and efficiencies of a hawkmoth-inspired flapping-wing model and a revolving-wing model with the same wings for $R e=50-4800$. They suggested that the lift force and the power are the most objective indices for comparing the performance. They concluded that the efficiency of the revolving-wing model declines rapidly with decrease of the Reynolds number. Consequently, for $R e \lesssim 100$, flapping wings are more advantageous than revolving wings.

The aformentioned comparative studies were for hovering flight. However, as Zheng et al. (2013) pointed out, it is difficult to apply results from hovering flight to situations such as forward flight or maneuvering. For flapping wings in free forward flight, the simple questions posed above have not been answered adequately. In the present study, we evaluate not only the lift force but also the aerodynamic power expenditure and the power loading of a revolving-wing model. We then compare this model with a butterfly-like model involving a body with flapping wings (referred to as a butterfly-like flapping wing-body model), which can fly freely both upward and forward (see Suzuki et al. 2015). In the butterfly-like flapping wing-body model, the wings flap downward 
to generate lift and backward to generate thrust, just like an actual butterfly. Therefore, this flapping wing-body model utilizes aerodynamic forces that are parallel to the wingtip path, i.e., drag-based forces (Ellington 1999, Ristroph et al. 2011). It has been reported that insects often use drag-based forces in rapid maneuvers such as sudden starts, stops, and turns (Ristroph et al. 2011). Thus, this flapping wing-body model incorporates important features of flapping flight by insects, namely free forward flight and maneuvering; these are also desirable features for MAVs.

In the present study, we attempt to evaluate the lift force, the power, and the power loading of a revolving-wing model. We use computational approach to seek answers to the above questions because we can easily and accurately compute moving-boundary flows around revolving and flapping wings thanks to recent advances in computational methods (e.g., Liu et al. 1998, Liu \& Kawachi 1998, Aono et al. 2008, Liu 2009). In recent years, various immersed boundary methods (IBMs) have been proposed to simulate moving-boundary flows in a Cartesian grid (see Mittal \& Iaccarino 2005). An IBM is a simple approach to moving-boundary flows, although certain techniques are necessary to satisfy the no-slip boundary condition at the moving boundary. In contrast, the lattice Boltzmann method (LBM) has been developed into an alternative and promising numerical scheme for simulating viscous fluid flows in a Cartesian grid without having to solve Poisson equation for the pressure field (see Succi 2001). Since both of these methods are Cartesian-based, the LBM combined with an IBM (referred to as IBLBM) is well suited to simulating moving-boundary flows. Several IB-LBM approaches have already been presented (e.g., Feng \& Michaelides 2005, Sui et al. 2008, Wu \& Shu 2009, Krüger et al. 2011) and applied to flows around flapping wings (e.g., De Rosis et al. 2014, Lee et al. 2015, Wu et al. 2015). Suzuki \& Inamuro (2011) also proposed an efficient IB-LBM for moving-boundary flows. This IB-LBM has been applied successfully to the flow and lift generated by a two-dimensional symmetric flapping wing (Ota et al. 2012, Kimura et al. 2014), a dragonfly-like flapping wing-body model (Minami et al. 2015), and a butterfly-like flapping wing-body model (Suzuki et al. 2015).

In the present paper, we perform numerical simulations using the IB-LBM proposed by Suzuki \& Inamuro (2011) to compare aerodynamic performance in relation to lift force, power, and power loading between a butterfly-like flapping wing-body model and a revolving-wing model. Both models have the same infinitely thin rectangular wings. We begin by calculating the aerodynamic performance of the flapping wing-body model for various kinematic parameters and of the revolving-wing model for various angles of attack for $R e=50-1000$. We then compare the aerodynamic performance of the two models. In the comparison, we consider not only the case in which the body of the flapping wing-body model is fixed but also the case in which it can move freely, i.e., free flight.

This paper is organized as follows. In section 2, we introduce the butterfly-like flapping wing-body model and the revolving-wing model. In section 3, we present the governing equations and the parameters of the systems. In section 4, we explain the computational method and conditions. In section 5, we define the aerodynamic 
performance indices. In section 6 , we present our results and discussions. Finally, in section 7 , we draw our conclusions.

\section{Wing models}

\subsection{Butterfly-like flapping wing-body model}

2.1.1. Components The butterfly-like flapping wing-body model proposed by Suzuki et al. (2015) is a simple wing-body model with infinitely thin square wings of side $L$ and a rod-shaped body of length $L_{\mathrm{b}}=L$. The flexibility and mass of the wings are neglected. The body has uniform (line) density $\rho_{\mathrm{b}}$. Therefore, the center of mass of the body is at its mid-point, and the total mass of the body is $M=\rho_{\mathrm{b}} L_{\mathrm{b}}$. The two wings are connected at the mid-points of the body and the wing root. It should be noted that the body has a negligible effect on the flow field and the aerodynamic forces acting on the model.

2.1.2. Wing motion The butterfly-like flapping wing-body model flaps downward and backward to generate lift and thrust forces, respectively. The motions of the two wings are symmetrical with respect to the longitudinal plane. The wing motion is a combination of an attacking motion and a flapping motion (see figure 1), described by

(a)

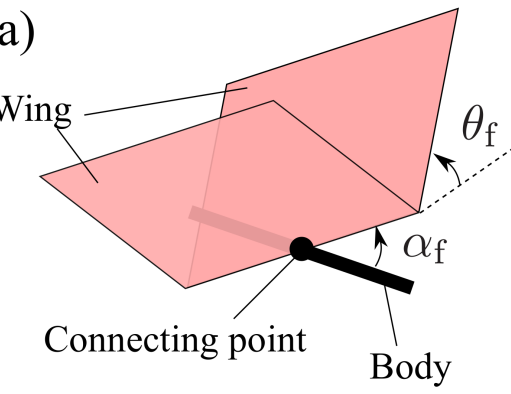

(c)

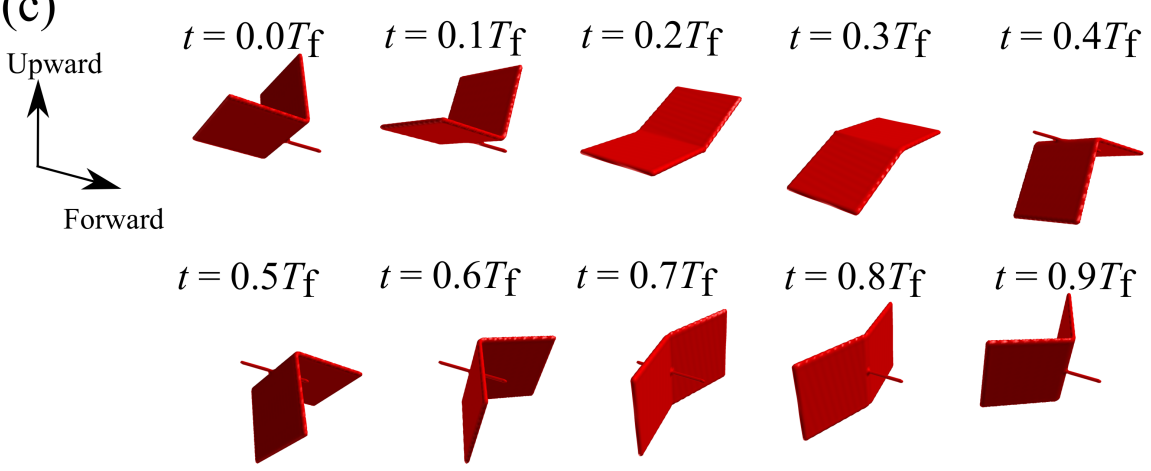

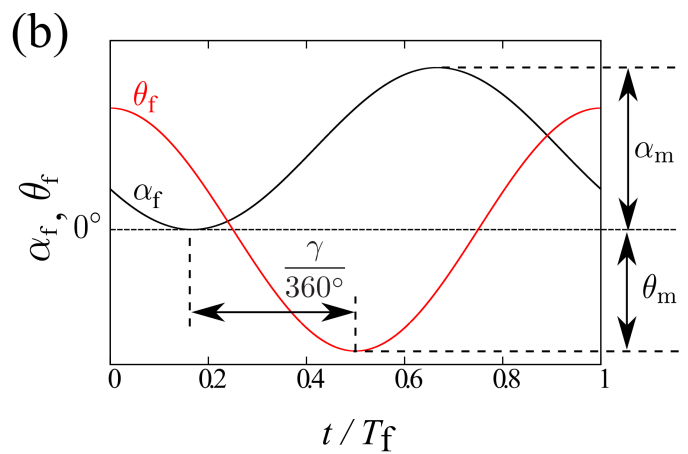

$t / T_{\mathrm{f}}$

Figure 1. (a) Perspective illustration of the butterfly-like flapping wing-body model. (b) Time variations of the angle of attack $\alpha_{\mathrm{f}}$ and the flapping angle $\theta_{\mathrm{f}}$ during one period. (c) Wing motion with $\alpha_{\mathrm{m}}=90^{\circ}, \theta_{\mathrm{m}}=45^{\circ}$, and $\gamma=90^{\circ}$ during one period. 
the angle of attack $\alpha_{\mathrm{f}}(t)$ and the flapping angle $\theta_{\mathrm{f}}(t)$, respectively, at time $t$ as follows:

$$
\begin{aligned}
& \alpha_{\mathrm{f}}(t)=\frac{\alpha_{\mathrm{m}}}{2}\left[1+\cos \left(\frac{2 \pi}{T_{\mathrm{f}}} t+\frac{\gamma}{180^{\circ}} \pi\right)\right], \\
& \theta_{\mathrm{f}}(t)=\theta_{\mathrm{m}} \cos \left(\frac{2 \pi}{T_{\mathrm{f}}} t\right),
\end{aligned}
$$

where $\alpha_{\mathrm{m}}$ is the maximum angle of attack, $\theta_{\mathrm{m}}$ is the flapping amplitude corresponding to half the stroke amplitude, $T_{\mathrm{f}}$ is the period of flapping motion, and $\gamma$ is the phase shift (see figure 1b). The wing motion of the butterfly-like flapping wing-body model is determined by the three parameters $\alpha_{\mathrm{m}}, \theta_{\mathrm{m}}$, and $\gamma$. The ranges of the maximum angle of attack, the flapping amplitude, and the phase shift are $0^{\circ} \leq \alpha_{\mathrm{m}} \leq 90^{\circ}, 0^{\circ}<\theta_{\mathrm{m}}<90^{\circ}$, and $0^{\circ} \leq \gamma \leq 180^{\circ}$, respectively. The model with $\alpha_{\mathrm{m}}=0^{\circ}$ flaps downward in the downstroke and upward in the upstroke symmetrically, whereas that with $\alpha_{\mathrm{m}}=90^{\circ}$ flaps downward in the downstroke and directly backward in the upstroke (see figure 1c). The range of the phase shift is determined so that the value of $\alpha_{\mathrm{f}}(t)$ becomes $0^{\circ}$ during the downstroke. If not, the model flaps not downward but forward in the downstroke. For more details about the wing motion of the flapping wing-body model, see Suzuki et al. (2015).

\subsection{Revolving wing model}

2.2.1. Components The revolving-wing model has the same components as the butterfly-like flapping wing-body model, i.e., two infinitely thin square wings of side $L$ and a rod-shaped body of length $L_{\mathrm{b}}=L$. The flexibility and the mass of the wings are neglected. It should be noted that the body has a negligible effect on the flow field and the aerodynamic forces acting on the model.

2.2.2. Wing motion In the revolving-wing model, the two wings revolve around the body at a constant angular velocity $\omega$ and with a constant angle of attack $\alpha_{\mathrm{r}}$ (see figure 2). The revolution period is given by $T_{\mathrm{r}}=2 \pi / \omega$. The angle of revolution for one wing at time $t$ is given by

$$
\theta_{\mathrm{r}}(t)=\omega t \text {. }
$$

The difference between the angles of revolution of the two wings is set to $180^{\circ}$, i.e., the angle of revolution of the other wing is $\omega t+\pi$.

\section{Governing equations}

\subsection{Fluid motion}

The fluid motion around the butterfly-like flapping wing-body model and the revolving wing model is governed by the continuity equation and the Navier-Stokes equation for an incompressible fluid:

$$
\boldsymbol{\nabla} \cdot \boldsymbol{u}=0
$$


(a)

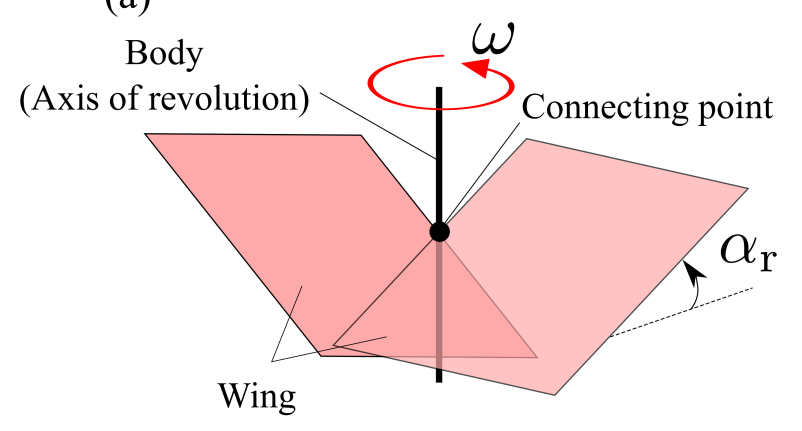

(b)

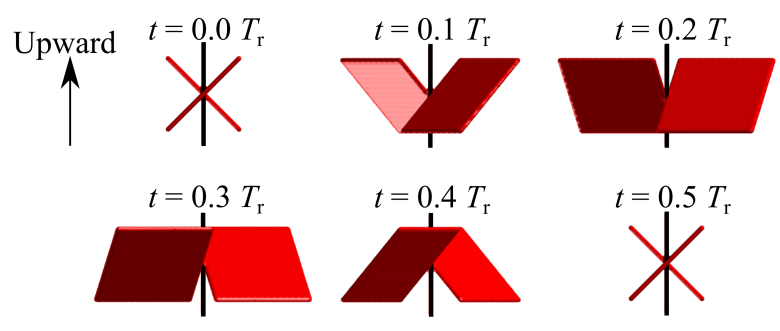

Figure 2. (a) Perspective illustration of the revolving-wing model with two rectangular wings. (b) Wing motion with $\alpha_{\mathrm{r}}=45^{\circ}$ during half a period as viewed from the right-hand side of the revolving-wing model.

$$
\frac{\partial \boldsymbol{u}}{\partial t}+(\boldsymbol{u} \cdot \nabla) \boldsymbol{u}=-\frac{1}{\rho_{\mathrm{f}}} \boldsymbol{\nabla} p+\nu \nabla^{2} \boldsymbol{u}
$$

where $\boldsymbol{u}$ is the fluid velocity, $p$ is the pressure, $\rho_{\mathrm{f}}$ is the density of the fluid, and $\nu$ is the kinematic viscosity of the fluid. We consider the fluid to be air at room temperature $\left(20^{\circ} \mathrm{C}\right)$, and we set $\rho_{\mathrm{f}}=1.205 \mathrm{~kg} / \mathrm{m}^{3}$ and $\nu=1.512 \times 10^{-5} \mathrm{~m}^{2} / \mathrm{s}$. The no-slip condition should be satisfied on the surfaces of the models, i.e., the fluid velocity must be equal to the velocity of the wings and the body.

The governing non-dimensional parameter of equations (4) and (5) is the Reynolds number, which is defined as:

$$
R e=\frac{U_{\mathrm{ref}} L}{\nu}
$$

where $U_{\text {ref }}$ is a characteristic flow speed, and $L$ is a characteristic length. For the latter, we take the wing length $L=18.1 \mathrm{~mm}$ of the small butterfly Janatella leucodesma (see Dudley 1990). For the former, we take the mean wing-tip speed defined by $U_{\text {tipf }}=4 \theta_{\mathrm{m}} L / T_{\mathrm{f}}$ as the characteristic flow speed for the butterfly-like flapping wingbody model, whereas we take the mean wing-tip speed defined by $U_{\text {tipr }}=\omega L$ as the characteristic flow speed for the revolving-wing model. In the following, we denote the Reynolds number with $U_{\text {ref }}=U_{\text {tipf }}$ as $R e_{\mathrm{f}}$ and that with $U_{\text {ref }}=U_{\text {tipr }}$ as $R e_{\mathrm{r}}$.

It should be noted that the flapping wing-body model has a totally different configuration to that of the revolving-wing model. Therefore, even if $R e_{\mathrm{f}}=R e_{\mathrm{r}}$, the two flows have no similarity. In addition, as Zheng et al. (2013) pointed out, the definition of $R e_{\mathrm{f}}$ is not unique. For example, one might consider that the Reynolds number 
based on the maximum tip speed is more appropriate. In this study, however, we compare the aerodynamic performance of the flapping wing-body model with that of the revolving-wing model when $R e_{\mathrm{f}}=R e_{\mathrm{r}}$ as a first comparison. In section 6, we discuss an appropriate way to compare the two models.

\subsection{Body motion}

As mentioned above, the mass of the wings is neglected in the models, and the aerodynamic forces generated by the wings act on the body through the connecting point. In the butterfly-like flapping wing-body model, the body motion is governed by the equation of motion incorporating the aerodynamic forces and the force due to gravity (see equation (3.5) in Suzuki et al. 2015). In contrast, the revolving-wing model is assumed to be hovering, i.e., the body of the wing model is fixed.

\section{Computational method and conditions}

The computational method used in this study is the same as that in Suzuki et al. (2015); we use the IB-LBM approach (Suzuki \& Inamuro 2011) to solve equations (4) and (5). For details of the numerical method, see Appendix A.

We use the same computational domain for both the butterfly-like flapping wingbody model and the revolving-wing model. The computational domain is a cube of side $W=12 L$ as shown in figure 3 . The $x-, y^{-}$, and $z$-axes are fixed to the domain, and we consider the directions of the $x$ - and $y$-axes to be the forward and upward directions, respectively. The periodic boundary condition is applied to the two sides perpendicular to the $x$-axis, and the no-slip boundary condition is applied to the other sides. The center of the body is fixed at height $H$ from the bottom of the domain. The domain is initially filled with a stationary fluid at uniform pressure. To save computational time, we use a multi-block grid (Inamuro 2012) that is composed of a fine grid of lattice spacing $\Delta x$ and a coarse grid of lattice spacing $2 \Delta x$. The inner fine grid is a cube of side $D=3 L$ whose center is placed at the center of the body, whereas the coarse grid is used in the rest of the domain. The spatial and temporal resolutions for each $R e_{\mathrm{f}, \mathrm{r}}$ are given in table 1 . It should be noted that the resolutions used for the butterfly-like flapping wing-body model are the same as those used by Suzuki et al. (2015). Table 1 includes the non-dimensional wing-tip speed $\hat{U}_{\text {tipf,r }}=U_{\text {tipf,r }} / c_{\mathrm{p}}\left(c_{\mathrm{p}}\right.$ is the particle speed $)$ and the relaxation time $\tau$ used in the lattice Boltzmann simulations (see Appendices A.1 and A.2).

\section{Definitions of aerodynamic performance}

Before showing computational results, we define the aerodynamic performance factors that are used to compare the flapping wing-body model and the revolving-wing model. 


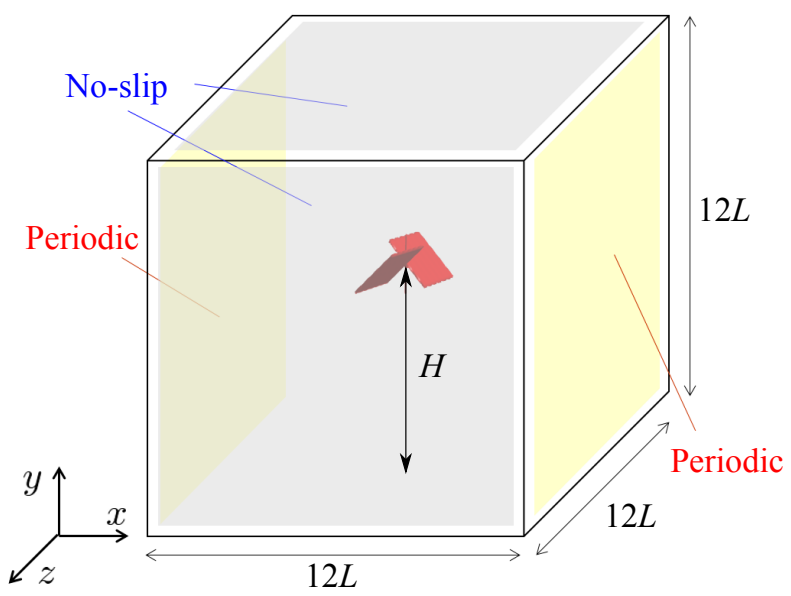

Figure 3. Computational domain for simulating flows around the butterfly-like flapping wing-body model and the revolving-wing model. The revolving-wing model is displayed in this figure as an example.

Table 1. Parameters used for the butterfly-like flapping wing-body model (left) and the revolving wing model (right).

\begin{tabular}{rcccc||rcccc}
\hline$R e_{\mathrm{f}}$ & $L$ & $T_{\mathrm{f}}$ & $\hat{U}_{\text {tipf }}$ & $\tau$ & $R e_{\mathrm{r}}$ & $L$ & $T_{\mathrm{r}}$ & $\hat{U}_{\text {tipr }}$ & $\tau$ \\
\hline 50 & $40 \Delta x$ & $6000 \Delta t$ & 0.021 & 0.5503 & 50 & $40 \Delta x$ & $6000 \Delta t$ & 0.042 & 0.6005 \\
100 & $40 \Delta x$ & $6000 \Delta t$ & 0.021 & 0.5251 & 100 & $40 \Delta x$ & $6000 \Delta t$ & 0.042 & 0.5503 \\
200 & $40 \Delta x$ & $6000 \Delta t$ & 0.021 & 0.5126 & 200 & $40 \Delta x$ & $6000 \Delta t$ & 0.042 & 0.5251 \\
300 & $50 \Delta x$ & $6000 \Delta t$ & 0.026 & 0.5139 & 300 & $50 \Delta x$ & $6000 \Delta t$ & 0.052 & 0.5262 \\
500 & $60 \Delta x$ & $6000 \Delta t$ & 0.031 & 0.5113 & 500 & $60 \Delta x$ & $6000 \Delta t$ & 0.063 & 0.5226 \\
1000 & $120 \Delta x$ & $12000 \Delta t$ & 0.031 & 0.5113 & 1000 & $120 \Delta x$ & $12000 \Delta t$ & 0.063 & 0.5226 \\
\hline
\end{tabular}

We calculate the lift force $F_{\mathrm{L}}$ and the power $P$ (which are often used as primitive aerodynamic performance factors) as follows:

$$
\begin{aligned}
& F_{\mathrm{L}}=\boldsymbol{F}_{\text {aero }} \cdot \boldsymbol{e}_{y}, \\
& P=\sum_{\text {wing }} \boldsymbol{f}_{\text {local }} \cdot \boldsymbol{u}_{\text {local }},
\end{aligned}
$$

where $\boldsymbol{F}_{\text {aero }}$ is the total aerodynamic force acting on the model, $\sum_{\text {wing }}$ means summation over the Lagrangian points on the wings, $\boldsymbol{f}_{\text {local }}$ is the force acting locally on the fluid at a Lagrangian point on a wing, and $\boldsymbol{u}_{\text {local }}$ is the fluid velocity at that point. Therefore, $P$ is the power expended in moving the wings against the aerodynamic forces. The IB-LBM calculations of equations (7) and (8) are given in Appendix A.5. Let the time-averaged values of $F_{\mathrm{L}}$ and $P$ over one stroke be $\overline{F_{\mathrm{L}}}$ and $\bar{P}$. We define the power loading (i.e., the lift force per unit power) as

$$
P L=\frac{\overline{F_{\mathrm{L}}}}{\bar{P}} \text {. }
$$

As often used in the evaluation of aerodynamic performance, the lift coefficient $C_{\mathrm{L}}$ 
and the power coefficient $C_{\mathrm{P}}$, which are non-dimensional forms of $F_{\mathrm{L}}$ and $P$, respectively, are also defined as follows:

$$
\begin{aligned}
C_{\mathrm{L}} & =\frac{F_{\mathrm{L}}}{0.5 \rho_{\mathrm{f}} U_{\text {ref }}^{2}\left(2 L^{2}\right)}, \\
C_{\mathrm{P}} & =\frac{P}{0.5 \rho_{\mathrm{f}} U_{\text {ref }}^{3}\left(2 L^{2}\right)} .
\end{aligned}
$$

Let the time-averaged values of $C_{\mathrm{L}}$ and $C_{\mathrm{P}}$ in one stroke be $\overline{C_{\mathrm{L}}}$ and $\overline{C_{\mathrm{P}}}$. We define the power-loading coefficient as follows:

$$
C_{\mathrm{PL}}=\frac{\overline{C_{\mathrm{L}}}}{\overline{C_{\mathrm{P}}}}=U_{\text {ref }} P L .
$$

It should be noted that the above non-dimensional values depend on the choice of the characteristic flow speed $U_{\text {ref. }}$ Let the non-dimensional values with $U_{\text {ref }}=U_{\text {tipf }}$ and with $U_{\text {ref }}=U_{\text {tipr }}$ have the subscripts "f" and "r," respectively, in the same way as for the Reynolds number. In recovering the dimensional values from the non-dimensional ones, we use the density and viscosity of air and the wing length of a small butterfly, as discussed in section 3.1.

\section{Results and discussion}

In this section, we show computational results of the aerodynamic performance of the butterfly-like flapping wing-body model and the revolving-wing model and compare their features. We also compare agility (usually regarded as an advantage of flapping flight) between the flapping wing-body model and the revolving-wing model.

\subsection{Aerodynamic performance of the butterfly-like flapping wing-body model}

Here, we calculate the aerodynamic performance of the butterfly-like flapping wingbody model for various maximum angles of attack $\alpha_{\mathrm{m}}$, flapping amplitudes $\theta_{\mathrm{m}}$, and phase shifts $\gamma$ when the body is fixed. We clarify representative parameter values for efficient lift.

Although the Reynolds number for a small butterfly is $R e_{\mathrm{f}} \simeq 1000$, we set $R e_{\mathrm{f}}=500$ here to save on computational cost. For the flapping wing-body model, Suzuki et al. (2015) showed that aerodynamic performance is relatively insensitive to Reynolds number for values of $R e$ greater than several hundred. In addition, the ground effect on the aerodynamic performance of the flapping wing-body model was also investigated by Suzuki et al. (2015), and the current height of $H=6 L$ is sufficient for the ground effect to be neglected.

Taking the basic set of parameters to be $\left(\alpha_{\mathrm{m}}, \theta_{\mathrm{m}}, \gamma\right)=\left(90^{\circ}, 45^{\circ}, 90^{\circ}\right)$, we fix two of the parameters while changing the third to investigate the effect of the latter on

the aerodynamic performance. Figure 4 shows the time-averaged lift coefficient $\overline{C_{\mathrm{Lf}}}$, the time-averaged power coefficient $\overline{C_{\mathrm{Pf}}}$ in the tenth stroke $\left(9 \leq t / T_{\mathrm{f}} \leq 10\right)$, and power-loading coefficient $C_{\text {PLf }}$ against each parameter. It can be seen from figure $4(\mathrm{a})$ 

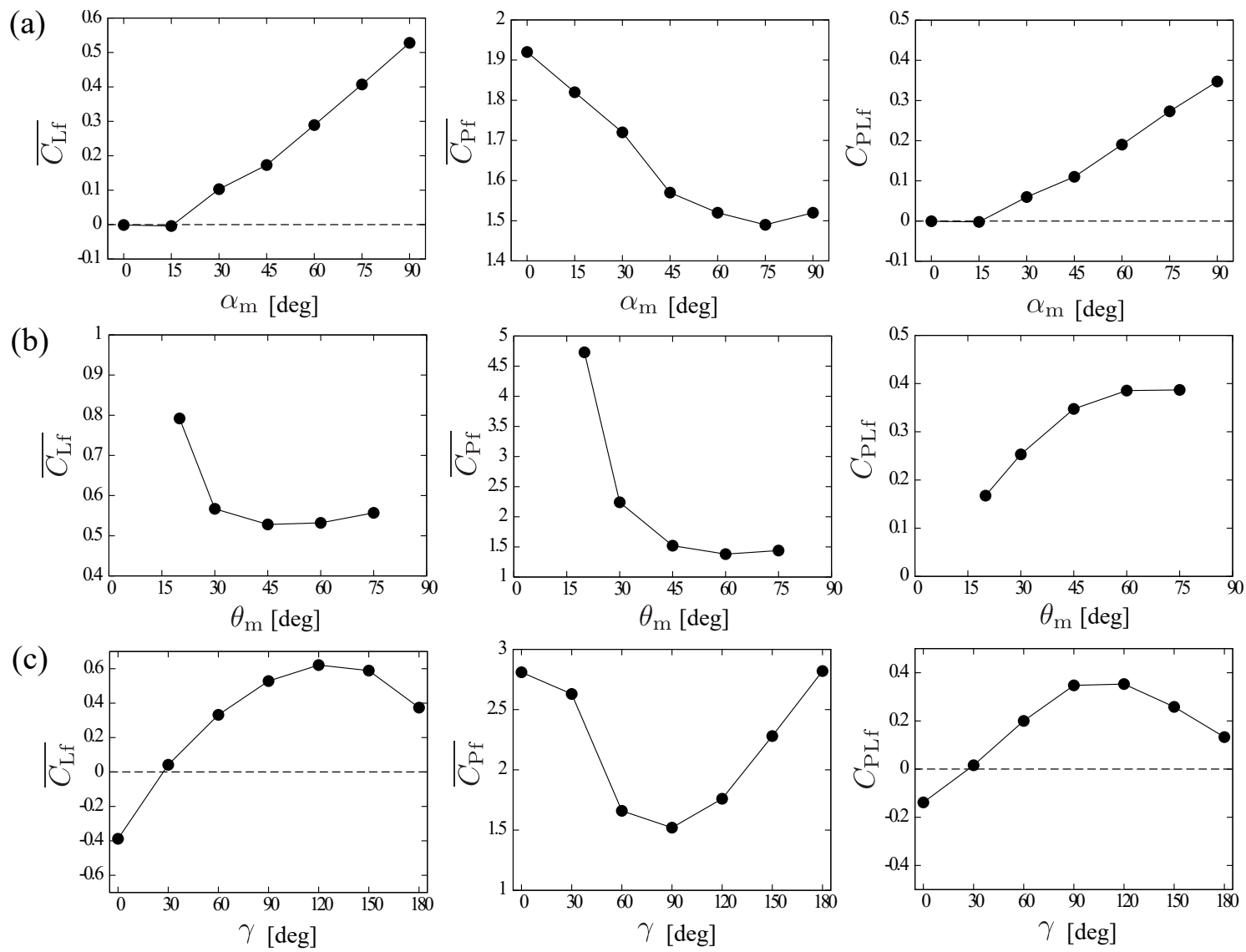

Figure 4. Time-averaged lift coefficient $\overline{C_{\mathrm{Lf}}}$, time-averaged power coefficient $\overline{C_{\mathrm{Pf}}}$, and power-loading coefficient $C_{\text {PLf }}$ against (a) maximum angle of attack $\alpha_{\mathrm{m}}$ with $\theta_{\mathrm{m}}=45^{\circ}$ and $\gamma=90^{\circ}$, (b) flapping amplitude $\theta_{\mathrm{m}}$ with $\alpha_{\mathrm{m}}=90^{\circ}$ and $\gamma=90^{\circ}$, and (c) phase shift $\gamma$ with $\alpha_{\mathrm{m}}=90^{\circ}$ and $\theta_{\mathrm{m}}=45^{\circ}$, all for $\operatorname{Re}_{\mathrm{f}}=500$.

that the time-averaged lift coefficient $\overline{C_{\mathrm{Lf}}}$ increases with the maximum angle of attack $\alpha_{\mathrm{m}}$, whereas the time-averaged power coefficient $\overline{C_{\mathrm{Pf}}}$ decreases with $\alpha_{\mathrm{m}}$. Therefore, the power-loading coefficient $C_{\mathrm{PLf}}$ increases with $\alpha_{\mathrm{m}}$, and $\alpha_{\mathrm{m}}=90^{\circ}$ is optimal in the butterfly-like flapping wing-body model. From figure $4(\mathrm{~b})$, we can see that $\overline{C_{\mathrm{Lf}}}$ and $\overline{C_{\mathrm{Pf}}}$ decrease with $\theta_{\mathrm{m}}$ in the range of $0<\theta_{\mathrm{m}}<45^{\circ}$ and then remain approximately steady for $\theta_{\mathrm{m}} \geq 45^{\circ}$. Consequently, $C_{\mathrm{PLf}}$ becomes almost optimal for $\theta_{\mathrm{m}} \geq 45^{\circ}$. From figure $4(\mathrm{c})$, we can see that $\overline{C_{\mathrm{Lf}}}$ is maximal for $\gamma=120^{\circ}, \overline{C_{\mathrm{Pf}}}$ is minimal for $\gamma=90^{\circ}$, and the resultant $C_{\mathrm{PLf}}$ is maximal for $\gamma=90^{\circ}$. From these results, we conclude that the parameter set $\left(\alpha_{\mathrm{m}}, \theta_{\mathrm{m}}, \gamma\right)=\left(90^{\circ}, 45^{\circ}, 90^{\circ}\right)$ is effectively optimal for the butterfly-like flapping wing-body model.

Here, we consider why the lift force can be generated efficiently with the above set of parameters. Figure 5 shows the time variation of the vortex structure near the flapping wing-body model for $\alpha_{\mathrm{m}}=90^{\circ}, \theta_{\mathrm{m}}=45^{\circ}$, and $\gamma=90^{\circ}$. The vortex structure is visualized using the Q-criterion (Hunt et al. 1988), i.e., the second invariant of the 
velocity-gradient tensor:

$$
Q=-\frac{\partial u_{i}}{\partial x_{j}} \frac{\partial u_{j}}{\partial x_{i}}
$$

where $i, j=x, y, z$ represent Cartesian coordinates and the summation convention is used. From figure 5, the change in vortex structure can be described as follows.

(i) A leading-edge vortex (LEV) and a wing-tip vortex (WTV) are generated on the upper surfaces of the wings in the early stage of a downstroke.

(ii) These vortices are then shed from the wing tip and move together down the upper surfaces of the wings in the later stage of the same downstroke.

(iii) The vortices are then released from the wings in the early stage of the subsequent upstroke.

(iv) The vortices are advected downward in the later stage of the same upstroke.

Like an actual butterfly (Yokoyama et al. 2013), the LEV and WTV on each wing upper surface are considered to be primary sources of lift for the flapping wing-body model. In addition, the aerodynamic performance depends on the generated LEV and WTV being released and advected downward without interference from the wings. This is because any such interference would weaken the downward flow induced under the model and strengthen the resisting force acting against the wings, and consequently the lift would decrease and the power expenditure would increase. Actually, in the case of
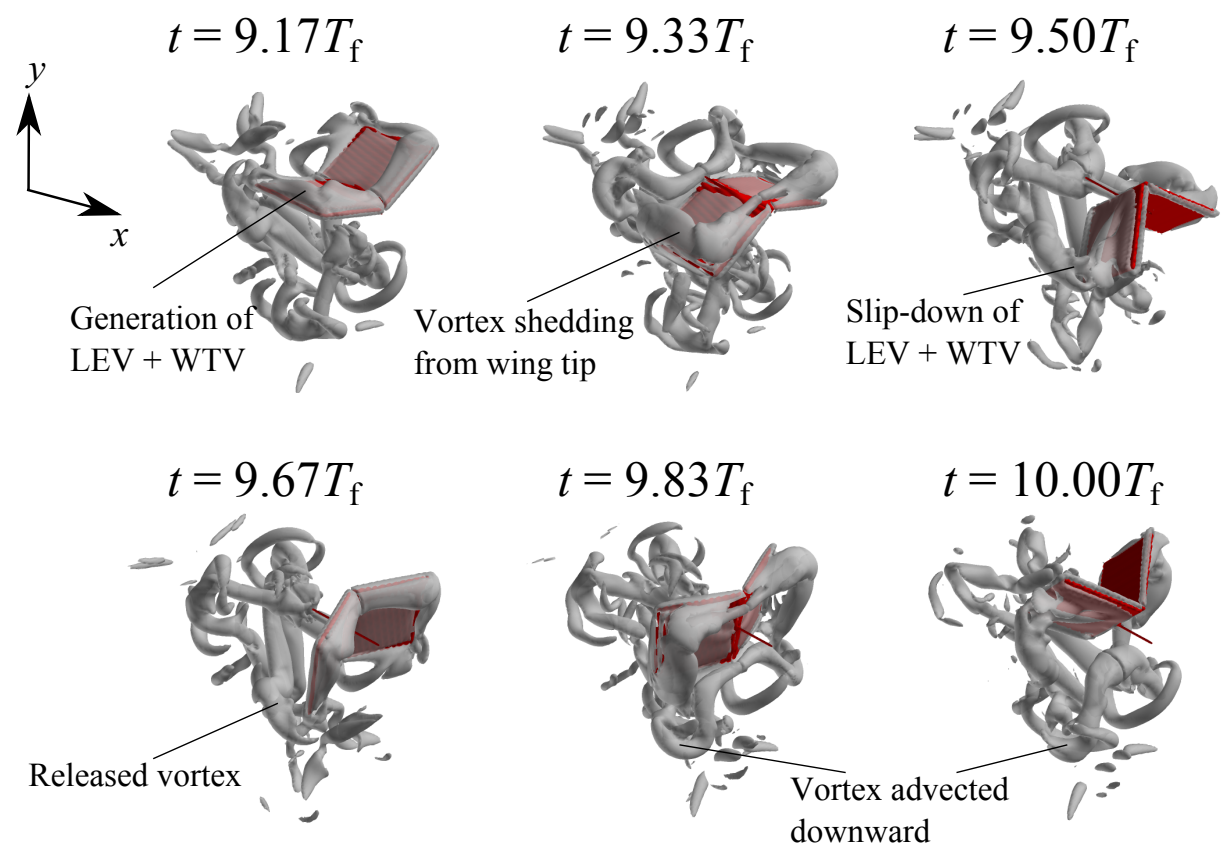

Figure 5. Time variations of the vortex structure visualized by the Q-criterion for the butterfly-like flapping wing-body model with $\alpha_{\mathrm{m}}=90^{\circ}, \theta_{\mathrm{m}}=45^{\circ}$, and $\gamma=90^{\circ}$ in the tenth cycle for $R e_{\mathrm{f}}=500$. The wing-body model is shown in red, and the isosurface of $Q=15\left(U_{\text {tipf }} / L\right)^{2}$ is shown in grey. 
a set of inferior parameter values, such interference occurs, or the LEV and WTV are shed before they grow sufficiently. Therefore, we consider the optimal parameter set $\alpha_{\mathrm{m}}=90^{\circ}, \theta_{\mathrm{m}}=45^{\circ}$, and $\gamma=90^{\circ}$ to be representative of the butterfly-like flapping wing-body model.

\subsection{Aerodynamic performance of the revolving-wing model}

Here, we calculate the aerodynamic performance of the revolving-wing model. In the following, we set the height of the revolving-wing model from the ground to $H=6 L$, and then examine the ground effect by changing the value of $H$. As noted in section 6.2.3, $H=6 L$ is sufficient for the ground effect to be neglected. We calculate the flow around the revolving-wing model for various angles of attack and for $50 \leq R e_{\mathrm{r}} \leq 1000$.

6.2.1. Aerodynamic performance for various angles of attack and Reynolds numbers Firstly, we consider the aerodynamic performance of the revolving-wing model. Figure 6 shows the time variations of the lift coefficient $C_{\mathrm{Lr}}$ and power coefficient $C_{\operatorname{Pr}}$ during $9 \leq t / T_{\mathrm{r}} \leq 10$ with $\alpha_{\mathrm{r}}=45^{\circ}$ for $R e_{\mathrm{r}}=50-1000$. We see from this figure that the lift coefficient increases and the power coefficient decreases with the Reynolds number. These results are consistent with the findings of Lentink \& Dickinson (2009b) and Zheng et al. (2013). Although the time variation becomes larger with the Reynolds number and a steady state is not achieved even at $t=10 T_{\mathrm{r}}$, we can see clear differences in the time-averaged values with the Reynolds number.
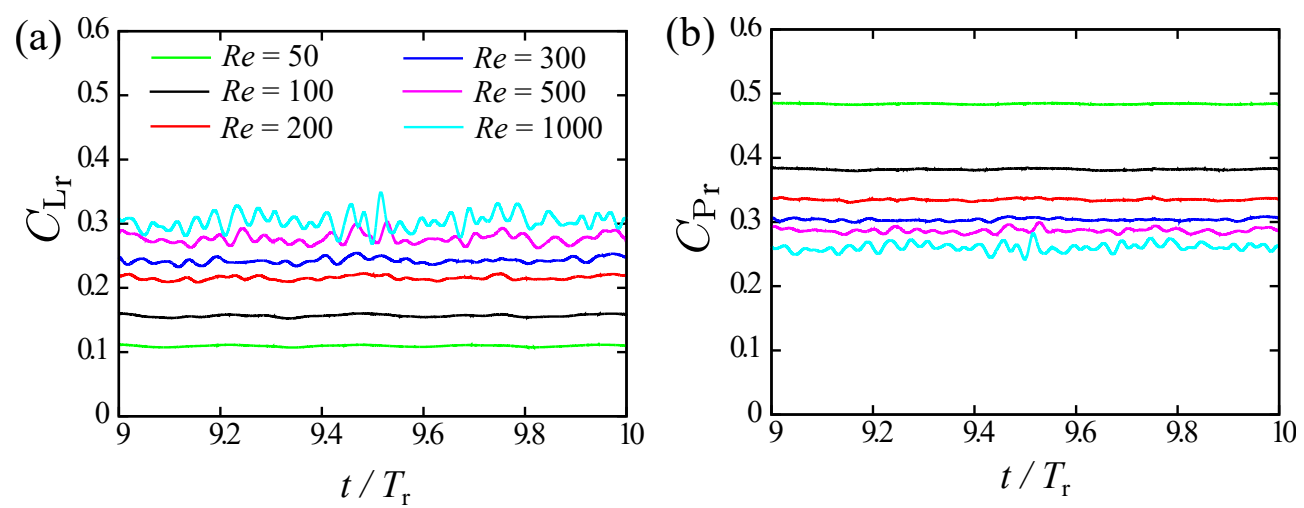

Figure 6. Time variations of (a) the lift coefficient $C_{\mathrm{Lr}}$ and (b) the power coefficient $C_{\text {Pr with }} \alpha_{\mathrm{r}}=45^{\circ}$ for $R e_{\mathrm{r}}=50-1000$.

Figure 7 shows the time-averaged lift coefficient $\overline{C_{\mathrm{Lr}}}$, the time-averaged power coefficient $\overline{C_{\operatorname{Pr}}}$ in the tenth stroke $\left(9 \leq t / T_{\mathrm{r}} \leq 10\right)$, and the power-loading coefficient $C_{\mathrm{PLr}}$. We can see from figure 7 (a) that, for any Reynolds number, the time-averaged lift coefficient $\overline{C_{\mathrm{Lr}}}$ increases with the angle of attack $\alpha_{\mathrm{r}}$ in the range of $\alpha_{\mathrm{r}}=10^{\circ}-60^{\circ}$, peaks around $\alpha_{\mathrm{r}}=60^{\circ}$, and decreases with $\alpha_{\mathrm{r}}$ in the range of $\alpha_{\mathrm{r}}=60^{\circ}-80^{\circ}$. For ordinary rotorcraft, however, the angle of attack for maximal $\overline{C_{\mathrm{Lr}}}$ is only a few degrees, i.e., much smaller than that for the present revolving-wing model. This is because the present 
(a)

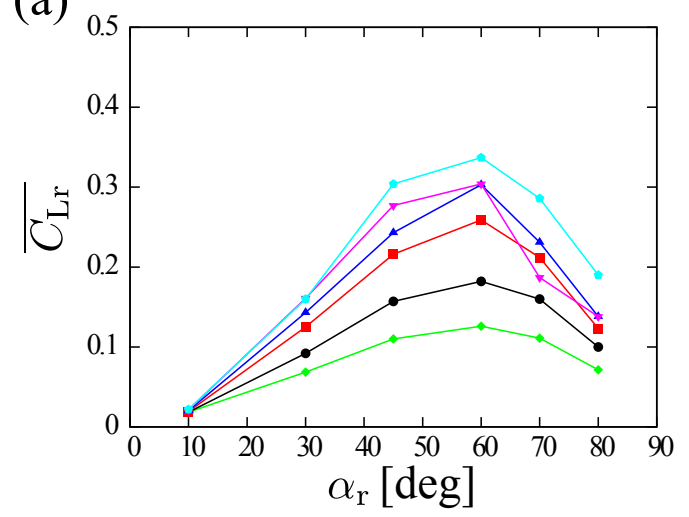

(c)

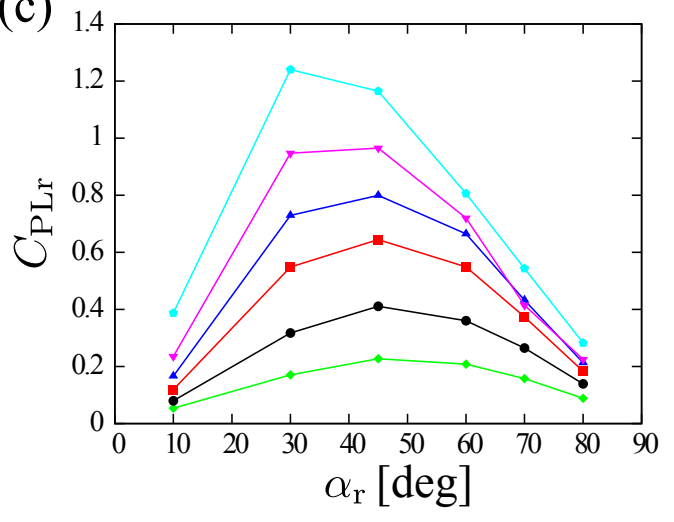

(b)

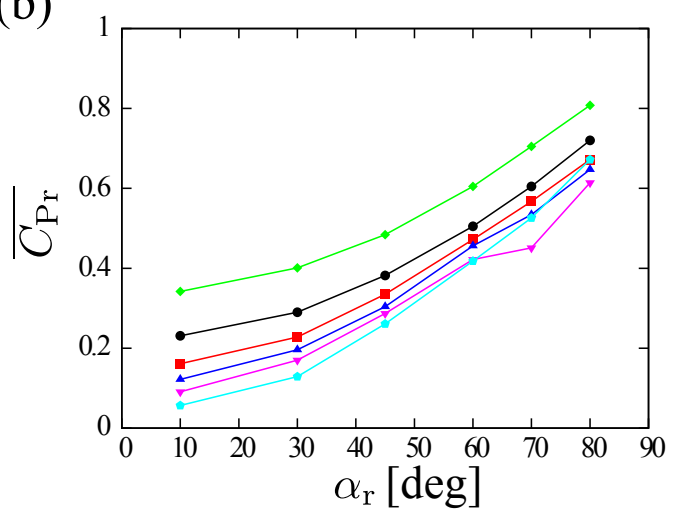

Figure 7. (a) Time-averaged lift coefficient $\overline{C_{\mathrm{Lr}}}$, (b) time-averaged power coefficient $\overline{C_{\mathrm{Pr}}}$, and (c) power-loading coefficient $C_{\mathrm{PLr}}$ against angle of attack $\alpha_{\mathrm{r}}$ for $R e_{\mathrm{r}}=50$ 1000 .

aspect ratio and Reynolds number are much lower than those for ordinary rotorcraft. Consequently, the effects of the wing tip and the viscosity are significant for the present revolving-wing model. This is consistent with the experimental results of Usherwood \& Ellington $(2002 a)$ that showed that a revolving hawkmoth wing generates its maximal lift force between $40^{\circ}$ and $50^{\circ}$. In addition, we can see from figure 7 (a) that, for any angle of attack except $\alpha_{\mathrm{r}}=70^{\circ}$, the time-averaged lift coefficient $\overline{C_{\mathrm{Lr}}}$ increases with the Reynolds number. The reason for the minor aberration in this overall trend at $R e_{\mathrm{r}} \approx 500$ for $\alpha_{\mathrm{r}}=70^{\circ}$ is not understood completely, but it is presumably due to the secondary vortices that appear under the wings as discussed in the next section.

From figure $7(\mathrm{~b})$, we can see that the time-averaged power coefficient $\overline{C_{\mathrm{Pr}}}$ decreases with the Reynolds number, and increases with the angle of attack. This is because the viscous skin friction decreases with the Reynolds number, and the drag force increases with the angle of attack. We can see from figure 7(c) that the power-loading coefficient $C_{\mathrm{PLr}}$ increases with the Reynolds number, and peaks around $\alpha_{\mathrm{r}}=45^{\circ}$.

6.2.2. Flow induced by the revolving-wing model Next, we consider the flow that is induced around the revolving-wing model. Figure 8 shows corresponding twodimensional velocity fields in the $x-y$ plane that includes the axis of revolution at 
$t=10 T_{\mathrm{r}}$ for $R e_{\mathrm{r}}=200$ and 500 . We can see from figure 8 that downward flows, so-called downwashes, are induced for $\alpha_{\mathrm{r}}=45^{\circ}, 60^{\circ}$, and $70^{\circ}$. Lift force should be generated in response to the downwash. We can see from figure 8 (a) that, for $R e_{\mathrm{r}}=200$, the horizontal component of the downwash becomes relatively large as the angle of attack increases. This is because more fluid is swept by the wings and revolves with them as the angle of attack increases. Therefore, more fluid should be pushed away from the axis of revolution by the centrifugal force. In addition, the flow near the wings increases with the angle of attack. The reason for this is not yet understood completely, but the same tendency was reported by Ozen \& Rockwell (2012). These results mean that the angle of attack determines the direction and the magnitude of the downwash, and consequently it determines the lift force. We can see from figure 8(b) that, for $R e_{\mathrm{r}}=500$, secondary vortices appear under the wing model and generate an upward jet that can be expected to disturb the generation of lift somewhat. Actually, for $\alpha_{\mathrm{r}}=70^{\circ}$, the time-averaged
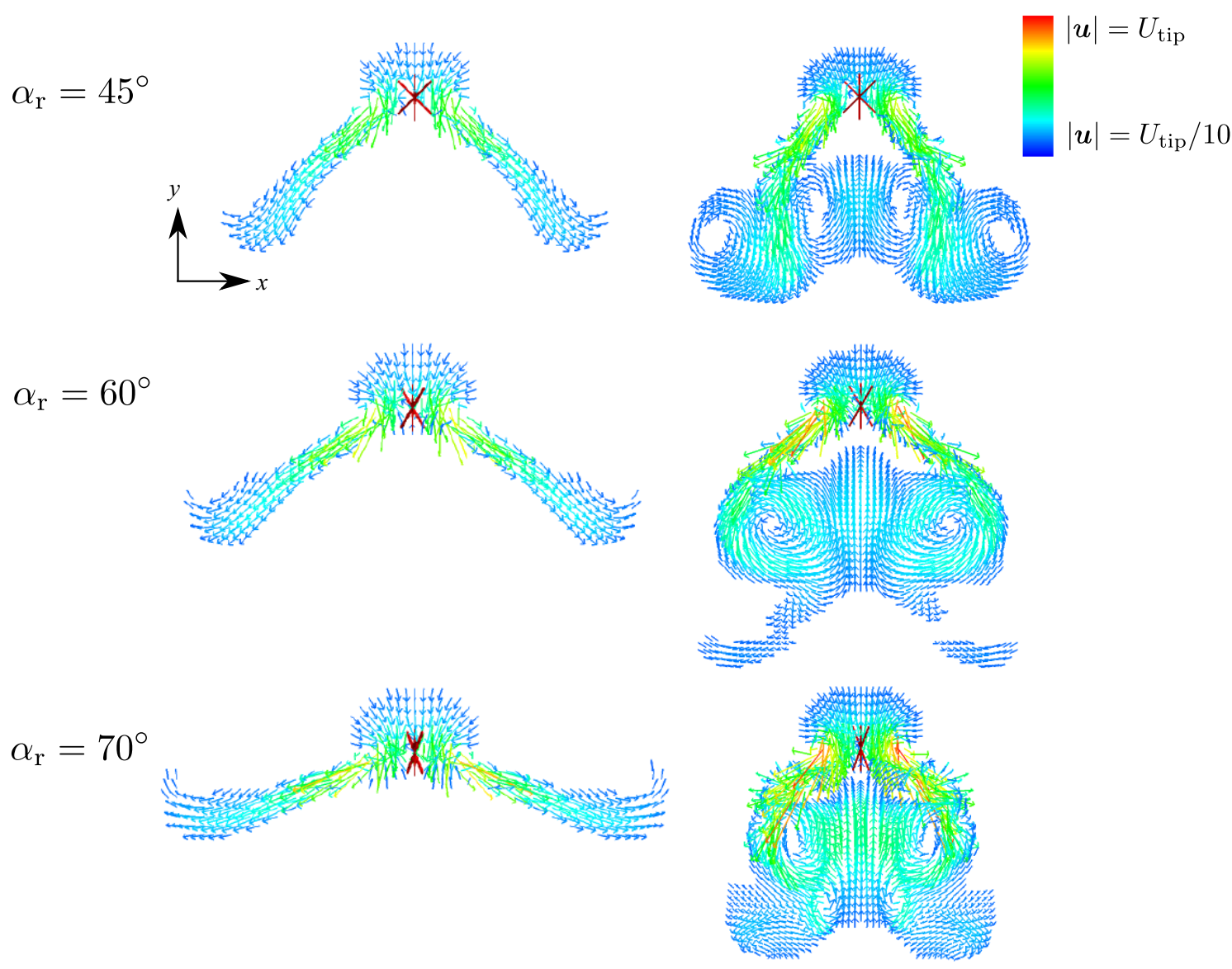

(a) $R e_{\mathrm{r}}=200$

(b) $R e_{\mathrm{r}}=500$

Figure 8. Two-dimensional velocity fields around the revolving-wing model for $\alpha_{\mathrm{r}}=45^{\circ}, 60^{\circ}$, and $70^{\circ}$ at $t=10 T_{\mathrm{r}}$ for (a) $R e_{\mathrm{r}}=200$ and (b) $R e_{\mathrm{r}}=500$ in the $x-y$ plane that includes the axis of revolution. The revolving-wing model is shown in red. The colors of the vectors denote the flow speed. The velocity vectors are drawn at every $8 \Delta x$. 

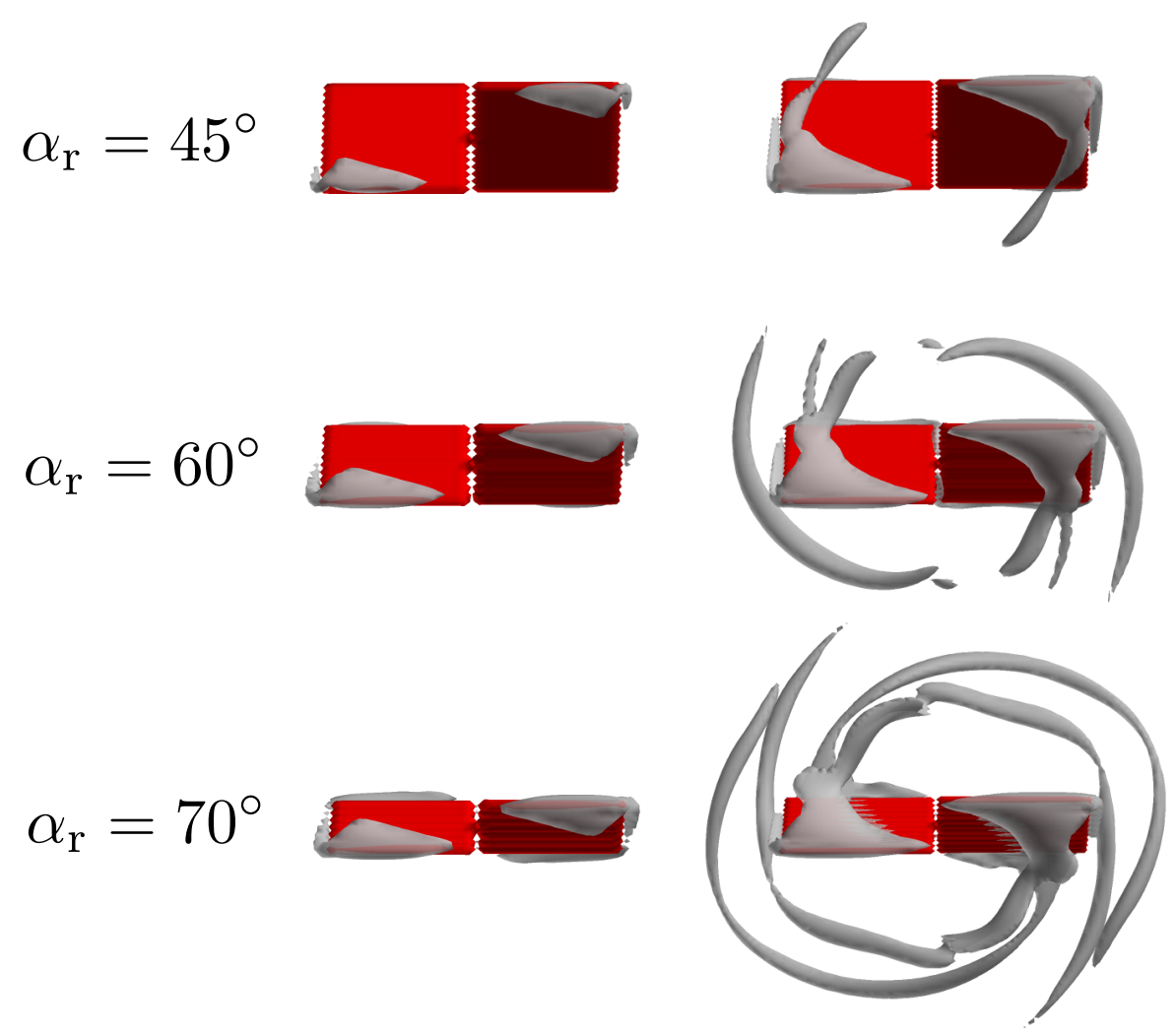
(a) $R e_{\mathrm{r}}=200$
(b) $R e_{\mathrm{r}}=500$

Figure 9. Vortex structure on revolving wings visualized by the Q-criterion for $\alpha_{\mathrm{r}}=45^{\circ}, 60^{\circ}$, and $70^{\circ}$ at (a) $R e_{\mathrm{r}}=200$ and (b) $R e_{\mathrm{r}}=500$. Views are from above in the steady state. The wing model is shown in red, and the isosurface of $Q=15\left(U_{\mathrm{tipr}} / L\right)^{2}$ is shown in grey.

lift coefficient for $R e_{\mathrm{r}}=500$ is smaller than that for $R e_{\mathrm{r}}=200$ (figure 7a), whereas it increases with the Reynolds number for the other values of $\alpha_{\mathrm{r}}$.

Figure 9 shows the vortex structure on the wings visualized by the Q-criterion for $\alpha_{\mathrm{r}}=45^{\circ}, 60^{\circ}$, and $70^{\circ}$ at $R e_{\mathrm{r}}=200$ and $R e_{\mathrm{r}}=500$. We can see from this figure that, even for a large angle of attack, the LEV is stably attached to the upper wing surfaces for both the Reynolds numbers. Many studies (e.g., Ozen \& Rockwell 2012) have reported that the LEV remains attached even for larger angles of attack at Reynolds numbers of order $10^{3}$. Because of this stable LEV, there is no sharp reduction in the lift coefficient as was seen in figure $7(\mathrm{a})$. In general, the magnitude of the net aerodynamic force increases as the LEV intensifies, and the vertical component of the net aerodynamic force (i.e., the lift force) decreases as the area of the wings projected onto the horizontal plane decreases. Therefore, the angle of attack that gives the maximal $\overline{C_{\mathrm{Lr}}}$ should be determined by a balance between the LEV intensity and the projected wing area. Comparing figures 9(a) and (b), we see that the LEV intensifies with the Reynolds 
number; for the high Reynolds number, it is shed near the wing tip while remaining attached to the leading edge. This is because viscous dissipation of vortices decreases with the Reynolds number. In addition, it can be seen from figure 9 (b) that the shed LEV tail lengthens with the angle of attack. It is reasonable that the LEV should separate easily at a high angle of attack, in the same way as the stall of an aircraft. The shed tail might be responsible for distorting the direction of the downwash and inducing the secondary vortex under the wing model at $R e_{\mathrm{r}}=500$, shown in figure 8(b). Moreover, the LEV structure on the model wings is similar to that on a hawkmoth's wings at an angle of attack of $45^{\circ}$, as reported by Zheng et al. (2013). In other words, even though the wing planforms are completely different, the LEV thickens toward the wing tips in both cases and its shed tail can be observed near the wing tip. Therefore, our results for the revolving-wing model are consistent with those of other studies.

6.2.3. Ground effect for the revolving-wing model Here, we examine the ground effect for the revolving-wing model. The ground effect for flapping wings has been investigated both experimentally (e.g., Zhang et al. 2014) and numerically (e.g., Gao \& Lu 2008, Maeda \& Liu 2013, Kolomenskiy et al. 2016), and it was reported that the ground effect for flapping wings in three-dimensional space is not significant. Indeed, Suzuki et al. (2015) showed that the ground effect is unimportant in simulations of the butterfly-like flapping wing-body model. From these previous studies, we expect the ground effect to be not particularly significant for the revolving-wing model as well. However, we should confirm this expectation for a fair comparison between the revolving-wing model and the flapping wing-body model.

Figure 10 shows $\overline{C_{\mathrm{Lr}}}, \overline{C_{\mathrm{Pr}}}$, and $C_{\mathrm{PLr}}$ against $H$ for $\alpha_{\mathrm{r}}=45^{\circ}$ and $R e_{\mathrm{r}}=500$. We can see from figure 10 (a) that whereas $\overline{C_{\mathrm{Lr}}}$ and $\overline{C_{\operatorname{Pr}}}$ are almost constant for $H \geq 6 L$, they increase as the model approaches the ground. However, such increases in $\overline{C_{\mathrm{Lr}}}$ and $\overline{C_{\mathrm{Pr}}}$ are less than $10 \%$ of the values for $H=6 \mathrm{~L}$. It can be seen from figure $10(\mathrm{~b})$ that $C_{\mathrm{PLr}}$ is almost independent of height $H$. This means that the ground effect has relatively little effect on the aerodynamic performance of the revolving-wing model.

\subsection{Comparison of the revolving-wing model and the butterfly-like flapping wing-body model}

As shown in sections 6.1 and 6.2, the maximum power loading for the butterfly-like flapping wing-body model is obtained around $\alpha_{\mathrm{m}}=90^{\circ}, \theta_{\mathrm{m}}=45^{\circ}$, and $\gamma=90^{\circ}$, and that for the revolving-wing model is obtained around $\alpha_{\mathrm{r}}=45^{\circ}$. In the following, we compare the two models at their respective maximum power loadings.

6.3.1. Comparison at the same Reynolds number We compare the time-averaged lift coefficient $\overline{C_{\mathrm{Lr}}}$, the time-averaged power coefficient $\overline{C_{\mathrm{Pr}}}$, and the power-loading coefficient $C_{\text {PLr }}$ of the revolving-wing model with those of the flapping wing-body model for $R e=R e_{\mathrm{f}}=R e_{\mathrm{r}}$, which means $U_{\mathrm{tipf}}=U_{\mathrm{tipr}}$ because the characteristic length and the 

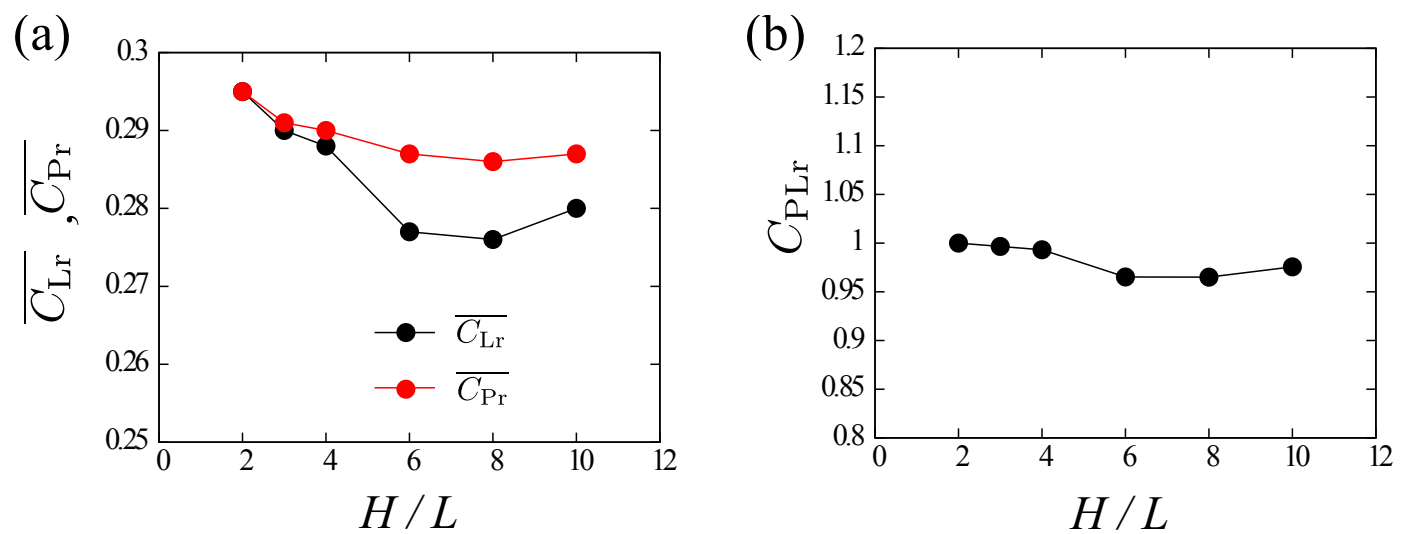

Figure 10. (a) Time-averaged lift and power coefficients $\overline{C_{\mathrm{Lr}}}$ and $\overline{C_{\mathrm{Pr}}}$ and (b) powerloading coefficient $C_{\mathrm{PLr}}$ against height $H$ of the revolving-wing model from the ground for $\alpha_{\mathrm{r}}=45^{\circ}$ and $R e_{\mathrm{r}}=500$.

kinematic viscosity are the same. As mentioned in section 3.1, the flows around flappingand revolving-wing models have no inherent similarity, even at the same Reynolds numbers. However, the flow speeds should be similar. Therefore, this comparison should be regarded as a comparison between two different wing models that induce flows with similar speeds.

Figure 11 shows the time-averaged lift and power coefficients and the power-loading coefficient against the Reynolds number. We see from figure 11(a) that the timeaveraged lift coefficient for the flapping wing-body model is roughly two times that for the revolving-wing model at the same Reynolds number. However, we see from figure 11(b) that the time-averaged power coefficient for the flapping wing-body model is roughly five times that for the revolving-wing model. This means that if the two models have the same wing-tip speed, the flapping wing-body model generates more lift but expends much more power than does the revolving-wing model. Consequently, as shown in figure 11(c), the power-loading coefficient for the flapping wing-body model is about a third of that for the revolving-wing model at $R e=1000$. Figures $11(\mathrm{~d}-\mathrm{f})$ shows the dimensional values of the lift force $\overline{F_{\mathrm{L}}}$, power $\bar{P}$, and power loading $P L$. We see that the power loading of the flapping wing-body model is much smaller than that of the revolving-wing model.

6.3.2. Comparison at the same power expenditure As mentioned in section 3 , the choice of characteristic flow speed for the flapping wing-body model is not unique. Because it appears in the lift coefficient, the power coefficient, and the Reynolds number, another characteristic flow speed might lead to significantly different results from the comparison shown in figure 11. Therefore, in order to compare different wing models, we should use indices that are independent of the choice of characteristic flow speed. One answer is to compare the dimensional lift force when the same dimensional power is expended, since the dimensional values do not require characteristic flow speed. Figure 12 shows the lift force $\overline{F_{\mathrm{L}}}$ and power loading $P L$ against the power $\bar{P}$. We can see from this figure 

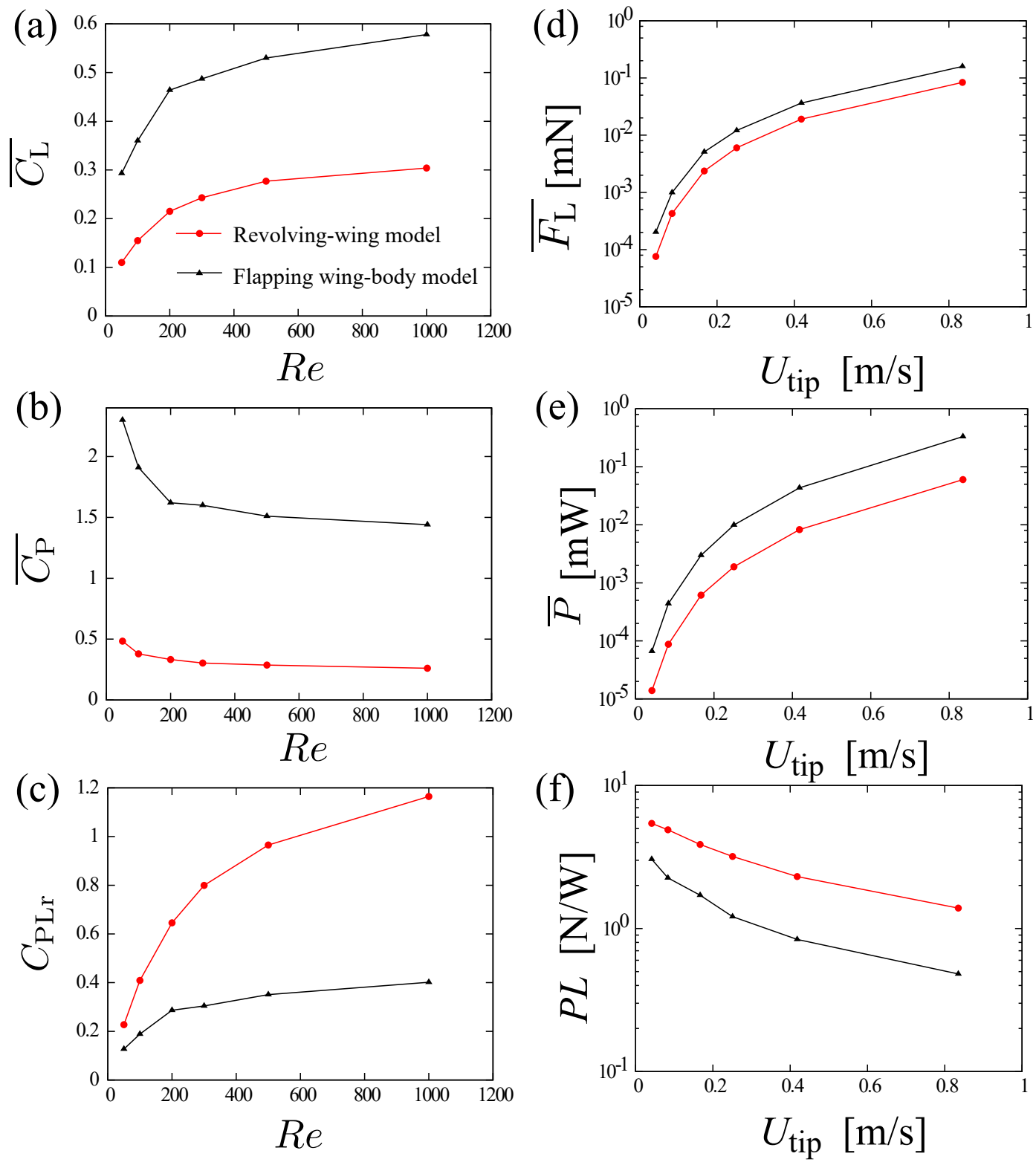

Figure 11. Comparisons of (a) time-averaged lift coefficient $\overline{C_{\mathrm{L}}}$, (b) time-averaged power coefficient $\overline{C_{\mathrm{P}}},(\mathrm{c})$ power-loading coefficient $C_{\mathrm{PL}},(\mathrm{d})$ time-averaged lift force $\overline{F_{\mathrm{L}}}$, (e) time-averaged power $\bar{P}$, and (f) power loading $P L$ at the same Reynolds number $R e=R e_{\mathrm{f}}=R e_{\mathrm{r}}$.

that, at the same power expenditure, the lift force and power loading of the flapping wing-body model are smaller than those of the revolving-wing model. This means for the same motor and battery, the revolving-wing model is the more efficient.

However, the above results are inconsistent with other comparable studies in which flapping wings are compared with revolving or fixed wings. Pesavento \& Wang (2009) compared a two-dimensional hovering flapping wing with a fixed wing in a steady flow, and concluded that flapping with optimal wing motion uses less power than does a fixed wing with optimal attack of angle if the flapping and fixed wings generate the same lift. 

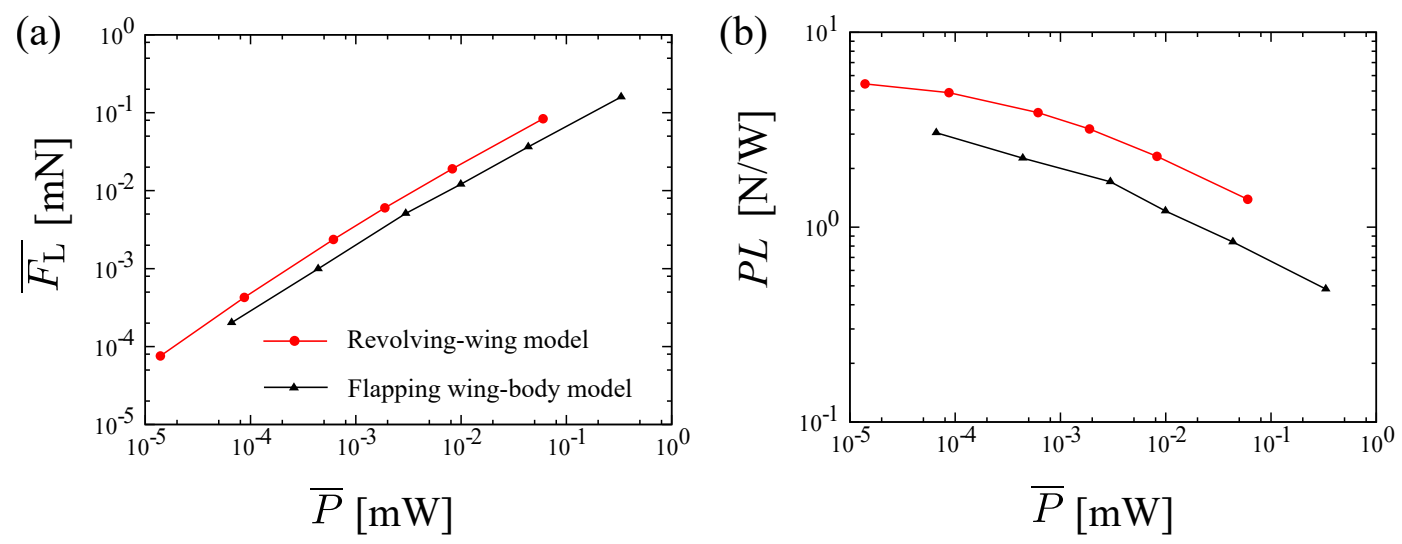

Figure 12. Comparisons of (a) lift force $\overline{F_{\mathrm{L}}}$ and (b) power loading $P L$ against power $\bar{P}$.

Zheng et al. (2013) reported that the lift and power expenditure for three-dimensional hovering flapping are comparable to those of revolving wings for the Reynolds numbers of an actual insect, and flapping is more advantageous than a revolving wing for $R e \lesssim 100$. The difference between the present results and those of Pesavento \& Wang (2009) is attributed partly to the dimensionality. The flow is confined to the longitudinal plane in two-dimensional simulations, whereas it can be induced additionally in the horizontal plane in three-dimensions. This horizontal flow might increase the power expenditure while contributing nothing to the lift. Another reason for the difference is considered to the drag-based thrust (Ellington 1999, Ristroph et al. 2011) of flapping. The lift force generated by hovering flapping, revolving, and fixed wings is perpendicular to the wingtip path. In contrast, butterfly-like flapping utilizes the aerodynamic forces parallel to the wing-tip path, called drag-based thrust. Since insects use drag-based thrust in brisk maneuvers (Ellington 1999), the butterfly-like flapping wing-body model might generate greater lift at the expense of efficiency. This is also a reason for the difference between the present results and those of Zheng et al. (2013).

It should be noted that the flapping wing-body model can generate thrust as well as lift. However, we have compared not the net force but the lift force of the flapping wing-body model with that of the revolving-wing model in the present section. One might consider the present comparison to be unfair because the thrust is not taken into account. If we compare the net force, however, the conclusion of the present section is unchanged. We define the magnitude of the net force as

$$
\overline{F_{\text {net }}}=\rho_{\mathrm{f}} U_{\text {ref }}^{2} L^{2} \sqrt{{\overline{C_{\mathrm{L}}}}^{2}+{\overline{C_{\mathrm{T}}}}^{2}},
$$

where $\overline{C_{\mathrm{T}}}$ is the time-averaged thrust coefficient. A comparison of the net-force magnitudes is shown in figure 13, where we can see that even the net force of the flapping wing-body model is smaller than that of the revolving-wing model.

6.3.3. Comparison with the flapping wing-body model in free flight In the above discussions, we considered the aerodynamic performance of the flapping wing-body 


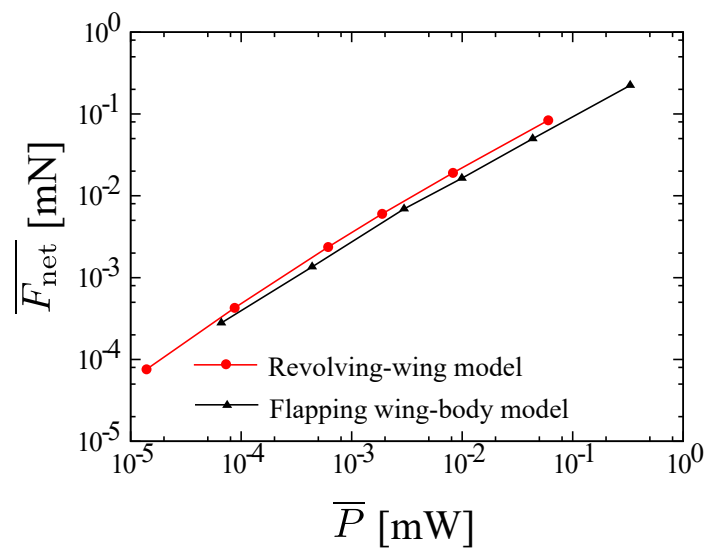

Figure 13. Comparison of net force $\overline{F_{\text {net }}}$ against power $\bar{P}$.

model when its body is fixed. The answer to the following simple question remains unclear. How does the aerodynamic performance of the flapping wing-body model change in free flight? Figure 14 shows the lift and power loading of the flapping wing-body model in free flight, for which it should be noted that the Froude number $F r=U_{\text {tipf }} / \sqrt{L G}$ ( $G$ is the gravitational acceleration) and the non-dimensional mass $N_{\mathrm{M}}=M /\left(\rho_{\mathrm{f}} L^{3}\right)$ ( $M$ is the mass of the body) are taken as the governing parameters in addition to the Reynolds number. However, because in the Earth's atmosphere and gravity the ratio of $F r$ to $R e_{\mathrm{f}}$ is determined by only the wing length $L$ (see Suzuki et al. 2015), set here as $L=18.1 \mathrm{~mm}$, the Froude number $F r$ is determined by the Reynolds number $R e_{\mathrm{f}}$. Therefore, it is enough to specify $\left(R e_{\mathrm{f}}, N_{\mathrm{M}}\right)$ to represent the results shown in figure 14 . When $\left(R e_{\mathrm{f}}, N_{\mathrm{M}}\right)=(1000,2.27)$ and $(1190,3.36)$ for $L=18.1 \mathrm{~mm}$, the flapping wing-body model can fly upward against gravity (see Suzuki et al. 2015). We can see from figure 14 that at the same power expenditure the lift and power loading in free flight are larger than those for a fixed body. This is because the lift increases because of the forward motion. However, in figures 14(a) and (b), the data for the flapping wing-body model in free flight appear to be under the extension of the revolving-wing curve. This means that although the flapping wing-body model in free flight can generate the lift more efficiently than when its body is fixed, the revolving-wing model is still more efficient.

\subsection{Discussion on agility}

Since the butterfly-like flapping wing-body model is a highly idealized model, the above discussions are not directly applied to the case of an actual butterfly. The wing planform, structure, and flexibility of butterflies might have evolved in order to achieve more efficient flight. In this section, however, we compare agility (which is regarded as an advantage of flapping insects) between the flapping wing-body model and the revolvingwing model. Although the present flapping wing-body model is highly idealized and far from an actual butterfly, we believe that this discussion provides a suggestion for understanding the flapping flight of actual butterflies. 

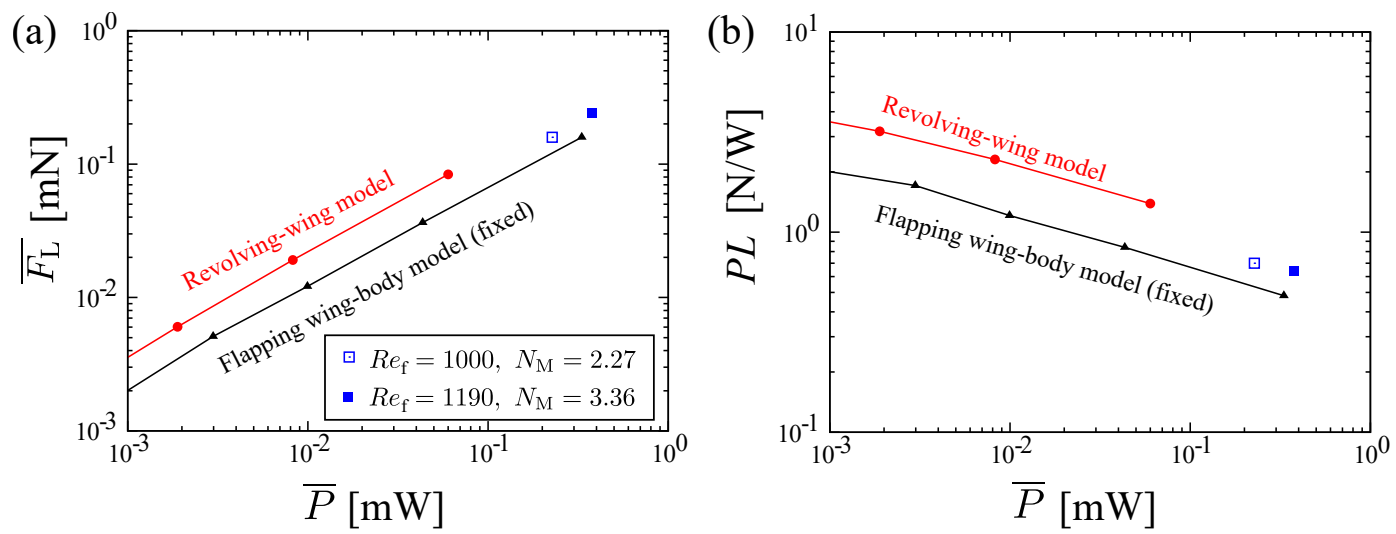

Figure 14. (a) Lift force $\overline{F_{\mathrm{L}}}$ and (b) power loading $P L$ against power $\bar{P}$ for the flapping wing-body model in free flight with $\left(R e_{\mathrm{f}}, N_{\mathrm{M}}\right)=(1000,2.27)$ and $(1190,3.36)$. This figure includes the results for the revolving-wing model and the flapping wing-body model when its body is fixed, which are the same as the data in figure 12 .

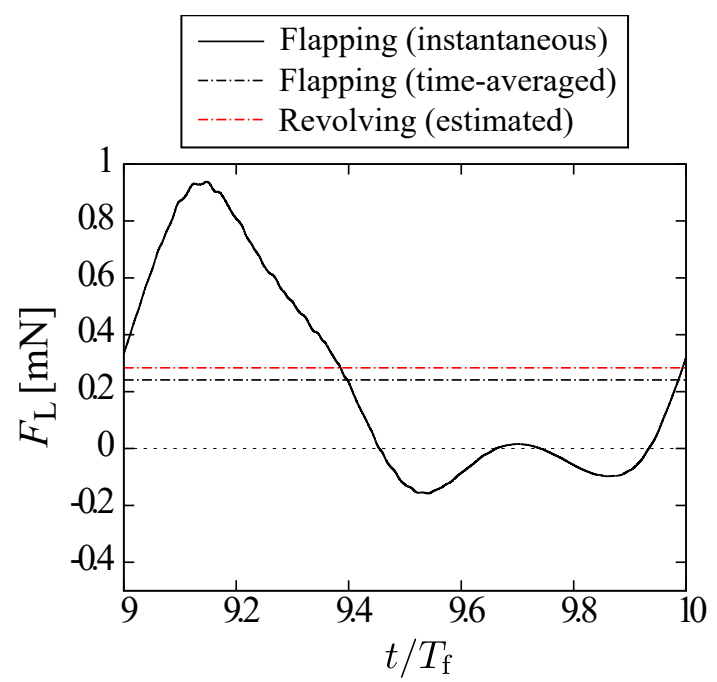

Figure 15. Comparison of instantaneous lift and time-averaged lift of the flapping wing-body model, and the estimated lift of the revolving-wing model at the same power expenditure of $\bar{P}=3.75 \times 10^{-1} \mathrm{~mW}$.

In the free flight of the flapping wing-body model under conditions of a small butterfly (i.e., $R e_{\mathrm{f}}=1190$ and $N_{\mathrm{M}}=3.36$ ), the model generates lift of $\overline{F_{\mathrm{L}}}=2.41 \times 10^{-1}$ $\mathrm{mN}$ at power expenditure of $\bar{P}=3.75 \times 10^{-1} \mathrm{~mW}$ averaged over one stroke. In contrast, the maximum lift during a stroke is $F_{\operatorname{Lmax}}=9.37 \times 10^{-1} \mathrm{mN}$. This value is about four times larger than the time-averaged lift (figure 15). Assuming that the lift and power coefficients of the revolving-wing model are unchanged for $R e_{\mathrm{r}}>1000$, we can estimate the dimensional lift force of the revolving-wing model as $\overline{F_{\mathrm{L}}}=2.84 \times 10^{-1} \mathrm{mN}$ at the same power expenditure as that of the flapping wing-body model. Therefore, the maximum lift of the flapping wing-body model is much larger than that of the revolvingwing model. Although agility refers in general to the rapidity of changes in speed and direction (Dudley 2002), a quantitative agility index is yet to be established. Because 
large instantaneous aerodynamic force induces a rapid change in speed, we can consider the magnitude of the instantaneous aerodynamic force an agility index. In this sense, the flapping wing-body model is more agile than the revolving-wing model. Such agility might be an advantage for actual butterflies in flight.

\section{Conclusions}

We have compared aerodynamic performance factors such as lift, power, and power loading of a butterfly-like flapping wing-body model with those of a revolving-wing model through numerical simulations using IB-LBM.

Firstly, we calculated the aerodynamic performance of the butterfly-like flapping wing-body model for various kinematic parameters, and that of the revolving-wing model for various angles of attack for Reynolds numbers in the range of 50-1000. For the flapping wing-body model, we found that the set of kinematic parameters $\left(\alpha_{\mathrm{m}}, \theta_{\mathrm{m}}, \gamma\right)=\left(90^{\circ}, 45^{\circ}, 90^{\circ}\right)$ is almost optimal in terms of lift efficiency. For the revolving-wing model, we found that the time-averaged lift coefficient $\overline{C_{\mathrm{Lr}}}$ peaks at an angle of attack of roughly $60^{\circ}$, whereas the time-averaged power coefficient $\overline{C_{\operatorname{Pr}}}$ increases with the angle of attack. Consequently, the power-loading coefficient $C_{\mathrm{PLr}}$ peaks at an angle of attack of roughly $45^{\circ}$. In addition, we found that the ground effect has little effect on the aerodynamic performance of the revolving-wing model.

We then compared the aerodynamic performance of the flapping wing-body model with that of the revolving-wing model at their respective maximal power loadings. For the same Reynolds number, we found that the flapping wing-body model generates more lift but expends much more power than does the revolving-wing model. Consequently, the power loading of the flapping wing-body model is much smaller than that of the revolving-wing model. As a more appropriate comparison, we compared the aerodynamic performance at the same power expenditure, and found that both the lift and power loading of the revolving-wing model are larger than those of the flapping wing-body model. Although the flapping wing-body model can generate lift more efficiently in free flight than when its body is fixed, the revolving-wing model is still more efficient. Finally, we found that the maximum lift of the flapping wing-body model is much larger than that of the revolving-wing model. This suggests that agility might be an advantage for actual butterflies in flight.

\section{Acknowledgements}

The authors would like to thank the anonymous reviewers for valuable comments and suggestions on the manuscripts. The authors would also like to thank Enago (www.enago.jp) for the English language review. 


\section{Appendix A. Immersed boundary-lattice Boltzmann method}

In this section, we describe the immersed boundary-lattice Boltzmann method (IBLBM) for solving the moving-boundary flows around the flapping wing-body model and the revolving-wing model.

\section{Appendix A.1. Non-dimensional variables for the IB-LBM}

We use the following non-dimensional variables defined by a characteristic length $H_{0}=L$, a characteristic particle speed $c_{\mathrm{p}}$, a characteristic time scale $t_{0}=H_{0} / U_{0}$ (where $U_{0}=U_{\text {tipf,r }}$ is a characteristic flow speed), and a fluid density $\rho_{\mathrm{f}}$ :

$$
\begin{array}{lll}
\hat{\boldsymbol{c}}_{i}=\boldsymbol{c}_{i} / c_{\mathrm{p}}, & \hat{\boldsymbol{x}}=\boldsymbol{x} / H_{0}, & \hat{t}=t / t_{0}, \\
\Delta \hat{x}=\Delta x / H_{0}, & \Delta \hat{t}=\Delta t / t_{0}, & \\
\hat{f}_{i}=f_{i} / \rho_{\mathrm{f}}, & \hat{\boldsymbol{u}}=\boldsymbol{u} / c_{\mathrm{p}}, & \hat{p}=p /\left(\rho_{\mathrm{f}} c_{\mathrm{p}}^{2}\right), \\
\hat{\nu}=\nu /\left(c_{\mathrm{p}} H_{0}\right), & \hat{\boldsymbol{g}}=\boldsymbol{g} H_{0} /\left(\rho_{\mathrm{f}} c_{\mathrm{p}}^{2}\right), & \\
\hat{\boldsymbol{X}}_{k}=\boldsymbol{X}_{k} / H_{0} & \hat{\boldsymbol{U}}_{k}=\boldsymbol{U}_{k} / c_{\mathrm{p}}, & \\
\hat{\boldsymbol{F}}_{\text {aero }}=\boldsymbol{F}_{\text {aero }} /\left(\rho_{\mathrm{f}} c_{\mathrm{p}}^{2} H_{0}^{2}\right), & \hat{P}=P /\left(\rho_{\mathrm{f}} c_{\mathrm{p}}^{3} H_{0}^{2}\right) . &
\end{array}
$$

Note that the circumflex represents "non-dimensional." It should be noted that the time step $\Delta t$ is equal to the time span during which the particles travel one lattice spacing, i.e., $\Delta x / \Delta t=c_{\mathrm{p}}$. We can easily obtain $\Delta \hat{t}=S h \Delta \hat{x}\left(\right.$ where $\left.S h=H_{0} /\left(t_{0} c_{\mathrm{p}}\right)=U_{0} / c_{\mathrm{p}}\right)$

from the above relation. Note that the circumflex in equation (A.1) is omitted in the following for simplicity.

\section{Appendix A.2. Lattice Boltzmann method}

In the LBM, we consider a model gas that is composed of identical particles whose velocities are restricted to a finite set of vectors (Succi 2001). A three-dimensional lattice with fifteen velocity vectors (D3Q15 model) is used in the present study. The D3Q15 model has the following velocity vectors:

$$
\begin{aligned}
& {\left[\boldsymbol{c}_{1}, \boldsymbol{c}_{2}, \boldsymbol{c}_{3}, \boldsymbol{c}_{4}, \boldsymbol{c}_{5}, \boldsymbol{c}_{6}, \boldsymbol{c}_{7}, \boldsymbol{c}_{8}, \boldsymbol{c}_{9}, \boldsymbol{c}_{10}, \boldsymbol{c}_{11}, \boldsymbol{c}_{12}, \boldsymbol{c}_{13}, \boldsymbol{c}_{14}, \boldsymbol{c}_{15}\right]=} \\
& {\left[\begin{array}{rrrrrrrrrrrrrrrr}
0 & 1 & 0 & 0 & -1 & 0 & 0 & 1 & -1 & 1 & 1 & -1 & 1 & -1 & -1 \\
0 & 0 & 1 & 0 & 0 & -1 & 0 & 1 & 1 & -1 & 1 & -1 & -1 & 1 & -1 \\
0 & 0 & 0 & 1 & 0 & 0 & -1 & 1 & 1 & 1 & -1 & -1 & -1 & -1 & 1
\end{array}\right] .}
\end{aligned}
$$

The evolution of the particle distribution function $f_{i}(\boldsymbol{x}, t)$ with the velocity $\boldsymbol{c}_{i}$ at the point $\boldsymbol{x}$ and time $t$ is computed by the following equations:

$$
f_{i}\left(\boldsymbol{x}+\boldsymbol{c}_{i} \Delta x, t+\Delta t\right)=f_{i}(\boldsymbol{x}, t)-\frac{1}{\tau}\left[f_{i}(\boldsymbol{x}, t)-f_{i}^{\mathrm{eq}}(p(\boldsymbol{x}, t), \boldsymbol{u}(\boldsymbol{x}, t))\right],
$$

where $\Delta x$ is a lattice spacing, $\Delta t$ is the time step during which the particles travel one lattice spacing, $f_{i}^{\text {eq }}$ is an equilibrium distribution function, $\tau$ is a relaxation time of $O(1)$, and $p(\boldsymbol{x}, t)$ and $\boldsymbol{u}(\boldsymbol{x}, t)$ are the pressure and the fluid velocity, respectively, given 
below. The equilibrium distribution function $f_{i}^{\text {eq }}$ of the incompressible model (He \& Luo 1997) is given by

$$
f_{i}^{\mathrm{eq}}(p, \boldsymbol{u})=E_{i}\left[3 p+3 \boldsymbol{c}_{i} \cdot \boldsymbol{u}+\frac{9}{2}\left(\boldsymbol{c}_{i} \cdot \boldsymbol{u}\right)^{2}-\frac{3}{2} \boldsymbol{u} \cdot \boldsymbol{u}\right],
$$

where $E_{1}=2 / 9, E_{2}=\cdots=E_{7}=1 / 9$, and $E_{8}=\cdots=E_{15}=1 / 72$. The pressure and the fluid velocity are calculated by

$$
\begin{aligned}
& p(\boldsymbol{x}, t)=\frac{1}{3} \sum_{i=1}^{15} f_{i}(\boldsymbol{x}, t), \\
& \boldsymbol{u}(\boldsymbol{x}, t)=\sum_{i=1}^{15} \boldsymbol{c}_{i} f_{i}(\boldsymbol{x}, t) .
\end{aligned}
$$

The asymptotic expansions of $\boldsymbol{u}$ and $p$ with respect to $\Delta x$ can be expressed by $\boldsymbol{u}=(\Delta x) \boldsymbol{u}^{(1)}+(\Delta x)^{2} \boldsymbol{u}^{(2)}+(\Delta x)^{3} \boldsymbol{u}^{(3)}+\cdots$ and $p=1 / 3+(\Delta x)^{2} p^{(2)}+(\Delta x)^{3} p^{(3)}+$ $(\Delta x)^{4} p^{(4)}+\cdots$, and $\boldsymbol{u}^{(1)}$ and $p^{(2)}$ satisfy the continuity equation (4) and the NavierStokes equation (5) with the kinematic viscosity $\nu$ given by

$$
\nu=\frac{1}{3}\left(\tau-\frac{1}{2}\right) \Delta x
$$

whereas $\boldsymbol{u}^{(2)}$ and $p^{(3)}$ are zero with appropriate initial and boundary conditions (Junk et al. 2005). Thus, the solutions of equations (A.3-A.6) give the pressure and the velocities for incompressible viscous fluid flows with relative errors of $O\left[(\Delta x)^{2}\right]$ (see Inamuro et al. 1997).

When an external body force $\boldsymbol{g}(\boldsymbol{x}, t)$ is applied, the evolution equation (A.3) of the particle distribution function $f_{i}(\boldsymbol{x}, t)$ is split into the following two steps:

$$
\begin{aligned}
& f_{i}^{*}\left(\boldsymbol{x}+\boldsymbol{c}_{i} \Delta x, t+\Delta t\right)=f_{i}(\boldsymbol{x}, t)-\frac{1}{\tau}\left[f_{i}(\boldsymbol{x}, t)-f_{i}^{\mathrm{eq}}(p(\boldsymbol{x}, t), \boldsymbol{u}(\boldsymbol{x}, t))\right], \\
& f_{i}(\boldsymbol{x}, t+\Delta t)=f_{i}^{*}(\boldsymbol{x}, t+\Delta t)+3 \Delta x E_{i} \boldsymbol{c}_{i} \cdot \boldsymbol{g}(\boldsymbol{x}, t+\Delta t) .
\end{aligned}
$$

\section{Appendix A.3. Immersed boundary method}

In the IBM, body forces are applied at lattice points near a boundary in order to enforce the no-slip condition on that boundary. The idea of the IBM was originally proposed by Peskin (1972) to simulate flows around flexible membranes in a Cartesian grid, and various IBMs have been proposed subsequently (see Mittal \& Iaccarino 2005). In this paper, we use the IBM proposed by Wang et al. (2008).

Supposing that $f_{i}(\boldsymbol{x}, t), \boldsymbol{u}(\boldsymbol{x}, t)$, and $p(\boldsymbol{x}, t)$ are known, the temporal $f_{i}^{*}(\boldsymbol{x}, t+\Delta t)$ and $\boldsymbol{u}_{i}^{*}(\boldsymbol{x}, t+\Delta t)$ can be calculated by equations (A.8) and (A.6), respectively. Let $\boldsymbol{X}_{k}(t+\Delta t)$ and $\boldsymbol{U}_{k}(t+\Delta t)(k=1,2, \ldots, N)$ be the Lagrangian points of the moving boundary and the boundary velocity at the points, respectively. Note that the moving boundary is represented by $N$ points, and the boundary Lagrangian points $\boldsymbol{X}_{k}$ generally 
differ from the Eulerian grid points $\boldsymbol{x}$. Then, the temporal velocities $\boldsymbol{u}_{i}^{*}\left(\boldsymbol{X}_{k}, t+\Delta t\right)$ at the boundary Lagrangian points $\boldsymbol{X}_{k}$ are interpolated by

$$
\boldsymbol{u}^{*}\left(\boldsymbol{X}_{k}, t+\Delta t\right)=\sum_{\boldsymbol{x}} \boldsymbol{u}^{*}(\boldsymbol{x}, t+\Delta t) W\left(\boldsymbol{x}-\boldsymbol{X}_{k}\right)(\Delta x)^{3},
$$

where $\sum_{\boldsymbol{x}}$ describes the summation over all lattice points $\boldsymbol{x}$, and $W$ is a weighting function proposed by Peskin (2002) and given by

$$
\begin{aligned}
& W(x, y, z)=\frac{1}{\Delta x} w\left(\frac{x}{\Delta x}\right) \cdot \frac{1}{\Delta x} w\left(\frac{y}{\Delta x}\right) \cdot \frac{1}{\Delta x} w\left(\frac{z}{\Delta x}\right), \\
& w(r)= \begin{cases}\frac{1}{8}\left(3-2|r|+\sqrt{1+4|r|-4 r^{2}}\right), & |r| \leq 1, \\
\frac{1}{8}\left(5-2|r|-\sqrt{-7+12|r|-4 r^{2}}\right), & 1 \leq|r| \leq 2, \\
0, & \text { otherwise. }\end{cases}
\end{aligned}
$$

The body force $\boldsymbol{g}(\boldsymbol{x}, t+\Delta t)$ is determined by the following iterative procedure.

Step 0. Compute the initial value of the body force at the boundary Lagrangian points by

$$
\boldsymbol{g}_{0}\left(\boldsymbol{X}_{k}, t+\Delta t\right)=\operatorname{Sh} \frac{\boldsymbol{U}_{k}-\boldsymbol{u}^{*}\left(\boldsymbol{X}_{k}, t+\Delta t\right)}{\Delta t},
$$

where it is noted that $S h / \Delta t=1 / \Delta x$ as defined in Appendix A.1.

Step 1. Compute the body force at the Eulerian grid points of the $\ell$ th iteration by

$$
\boldsymbol{g}_{\ell}(\boldsymbol{x}, t+\Delta t)=\sum_{k=1}^{N} \boldsymbol{g}_{\ell}\left(\boldsymbol{X}_{k}, t+\Delta t\right) W\left(\boldsymbol{x}-\boldsymbol{X}_{k}\right) \Delta V,
$$

where the body force is added not to one boundary Lagrangian point but to a small volume element whose volume is described as $\Delta V$. In this method, $\Delta V$ is taken as $S / N \times \Delta x$, where $S=2 L^{2}$ is the area of the surface of the wing model, and $S / N$ is taken to be approximately equal to $(\Delta x)^{2}$. It should be noted that whereas Peskin (2002) imposed $S / N<0.5(\Delta x)^{2}$ in order to avoid leaks, we found in our preliminary calculations that the results with $S / N$ in the range of $0.5(\Delta x)^{2}$ to $1.0(\Delta x)^{2}$ were almost coincident.

Step 2. Correct the velocity at the Eulerian grid points by

$$
\boldsymbol{u}_{\ell}(\boldsymbol{x}, t+\Delta t)=\boldsymbol{u}^{*}(\boldsymbol{x}, t+\Delta t)+\frac{\Delta t}{S h} \boldsymbol{g}_{\ell}(\boldsymbol{x}, t+\Delta t)
$$

Step 3. Interpolate the velocity at the boundary Lagrangian points with

$$
\boldsymbol{u}_{\ell}\left(\boldsymbol{X}_{k}, t+\Delta t\right)=\sum_{\boldsymbol{x}} \boldsymbol{u}_{\ell}(\boldsymbol{x}, t+\Delta t) W\left(\boldsymbol{x}-\boldsymbol{X}_{k}\right)(\Delta x)^{3} .
$$

Step 4. Correct the body force with

$$
\boldsymbol{g}_{\ell+1}\left(\boldsymbol{X}_{k}, t+\Delta t\right)=\boldsymbol{g}_{\ell}\left(\boldsymbol{X}_{k}, t+\Delta t\right)+S h \frac{\boldsymbol{U}_{k}-\boldsymbol{u}_{\ell}\left(\boldsymbol{X}_{k}, t+\Delta t\right)}{\Delta t},
$$

then return to Step 1.

From preliminary computations, we found that $\boldsymbol{g}_{\ell=5}(\boldsymbol{x}, t+\Delta t)$ is sufficient to maintain the no-slip condition on the boundaries. Therefore, we iterate the above procedure until $\ell=5$ in the present computations. 


\section{Appendix A.4. Boundary condition of the domain}

For the boundary of the computational domain (figure 3), we use periodic and no-slip boundary conditions. In the LBM, these boundary conditions have to be specified in terms of the particle distribution function.

The periodic boundary condition on the two sides perpendicular to the $x$-axis are satisfied as follows:

$$
\left\{\begin{array} { l } 
{ f _ { 2 } ^ { \text { left } } = f _ { 2 } ^ { \text { right } } , } \\
{ f _ { 8 } ^ { \text { left } } = f _ { 8 } ^ { \text { right } } , } \\
{ f _ { 1 0 } ^ { \text { left } } = f _ { 1 0 } ^ { \text { right } } , } \\
{ f _ { 1 1 } ^ { \text { left } } = f _ { 1 1 } ^ { \text { right } } , } \\
{ f _ { 1 3 } ^ { \text { left } } = f _ { 1 3 } ^ { \text { right } } , }
\end{array} \quad \left\{\begin{array}{l}
f_{5}^{\text {right }}=f_{5}^{\text {left }}, \\
f_{9}^{\text {right }}=f_{9}^{\text {left }}, \\
f_{12}^{\text {right }}=f_{12}^{\text {left }}, \\
f_{14}^{\text {right }}=f_{14}^{\text {left }}, \\
f_{15}^{\text {right }}=f_{15}^{\text {left }},
\end{array}\right.\right.
$$

where the superscripts "left" and "right" mean the left- and right-hand sides in figure 3, respectively. It should be noted that the right-hand sides in equation (A.18) can be calculated by the evolution equation (A.3) of the particle distribution functions.

The no-slip boundary condition on the other sides of the domain is satisfied by the bounce-back method (Succi 2001). In our preliminary calculation, however, when the relaxation time $\tau$ was smaller than about 0.53 , the calculation broke down from the walls at which the bounce-back method was applied. Although the reason for this is not yet understood, the slip velocity on the walls, which is negligible for small $\tau$ (see Inamuro et al. 1995), seemed to be the origin of the breakdown. In order to prevent it, we add a body force that eliminates the slip velocity in the same way as an immersed boundary method. For example, on the bottom wall of the domain, the temporal distribution functions are calculated by the bounce-back method:

$$
\left\{\begin{array}{l}
f_{3}^{*}=f_{6} \\
f_{8}^{*}=f_{12} \\
f_{9}^{*}=f_{13} \\
f_{11}^{*}=f_{15} \\
f_{14}^{*}=f_{10}
\end{array}\right.
$$

By using the above temporal distribution functions, we can calculate the temporal velocity $\boldsymbol{u}^{*}$ by equation (A.6), which should have an error from the no-slip boundary condition. In order to eliminate the error, we add a body force $\boldsymbol{g}=-\boldsymbol{u}^{*} S h / \Delta t$ to the wall. Therefore, the distribution functions on the wall are corrected by the body force by equation (A.9). With the above correction, the calculation does not break down even when the relaxation time $\tau$ is small.

\section{Appendix A.5. Calculation of aerodynamic performance}

The total aerodynamic force acting on the model is obtained by (Lai \& Peskin 2000)

$$
\boldsymbol{F}_{\text {aero }}(t)=-\sum_{\boldsymbol{x}} \boldsymbol{g}(\boldsymbol{x}, t)(\Delta x)^{3}
$$


where the internal mass effect (see Suzuki \& Inamuro 2011) is neglected, because the wing model has no volume. It should be noted that equation (A.20) includes the aerodynamic force and torque acting on not only the wings but also the body. However, in our preliminary calculations, the aerodynamic force acting on the body was two orders of magnitude smaller than that acting on the wings. Although the aerodynamic force acting on the body could be neglected, we choose to take it into account in this study. The aerodynamic power (8) is calculated by

$$
P(t)=\sum_{k=1}^{N} \boldsymbol{g}\left(\boldsymbol{X}_{k}, t\right) \cdot \boldsymbol{u}\left(\boldsymbol{X}_{k}, t\right) \Delta V .
$$

Appendix A.6. Accuracy of the method

The accuracy of the present method has been examined extensively through many benchmark problems of moving-boundary flows: an oscillating circular cylinder in a stationary fluid, the sedimentation of an elliptical cylinder, the sedimentation of a sphere, a flow around the almost impulsively moving plate, and flows around a flapping wing (Suzuki \& Inamuro 2011, Ota et al. 2012, Suzuki et al. 2015). Therefore, the present method should give accurate and reliable results for the flows around the butterfly-like flapping wing-body model and the revolving-wing model.

\section{References}

Aono H, Liang F \& Luo H 2008 J. Exp. Biol. 211, 239-257.

De Rosis A, Ubertini S \& Ubertini F 2014 J. Fluid Struct. 45, 202-215.

Dickinson M H, Lehman F O \& Sane S P 1999 Science 284, 1954-1960.

Dudley R 1990 J. Exp. Biol. 150, 37-53.

Dudley R 2002 Integ. Comp. Biol. 42, 135-140.

Ellington C P 1999 J. Exp. Biol. 202, 3439-3448.

Ellington C P, van den Berg C, Willmott A P \& Thomas A L R 1996 Nature 384, 626-630.

Feng Z G \& Michaelides E E 2005 J. Comput. Phys. 202, 20-51.

Gao T \& Lu X 2008 Phys. Fluids 20, 087101 (11pp).

Garmann D J \& Visbal M R 2014 J. Fluid Mech. 748, 932-956.

Garmann D J, Visbal M R \& Orkwis P D 2013 Phys. Fluids 25, 034101 (27pp).

Harbig R R, Sheridan J \& Thompson M C 2013a J. Fluid Mech. 730, 52-75.

Harbig R R, Sheridan J \& Thompson M C 2013b J. Fluid Mech. 717, 166-192.

He X \& Luo L S 1997 J. Stat. Phys. 88, 927-944.

Hunt J C R, Wray A A \& Moin P 1988 in 'Proc. of the Summer Program 1988' pp. 193-208.

Inamuro T 2012 Fluid Dyn. Res. 44, 024001 (21pp).

Inamuro T, Yoshino M \& Ogino F 1995 Phys. Fluids 7, 2928-2930; Erratum: 1996 8, 1124.

Inamuro T, Yoshino M \& Ogino F 1997 Phys. Fluids 9, 3535-3542.

Junk M, Klar A \& Luo L S 2005 J. Comput. Phys. 210, 676-704.

Kimura Y, Suzuki K \& Inamuro T 2014 Int. J. Mod. Phys. C 25, 1340020 (8pp).

Kolomenskiy D, Maeda M, Engels T, Liu H, Schneider K \& Nave J C 2016 PLOS ONE 11, e0152072 (21pp).

Krüger T, Varnik F \& Raabe D 2011 Comput. Math. Appl. 61, 3485-3505.

Lai M C \& Peskin C S 2000 J. Comput. Phys. 160, 705-719.

Lee J S, Seo I S \& Lee S H 2015 J. Fluids Struct. 54, 422-439. 
Lentink D \& Dickinson M H 2009a J. Exp. Biol. 212, 2691-2704.

Lentink D \& Dickinson M H $2009 b$ J. Exp. Biol. 212, 2705-2719.

Liu H 2009 J. Comput. Phys. 228, 439-459.

Liu H, Ellington C P, Kawachi K, van den Berg C \& Willmott A P 1998 J. Exp. Biol. 201, 461-477.

Liu H \& Kawachi K 1998 J. Comput. Phys. 146, 124-156.

Ma K Y, Chirarattananon P, Fuller S B \& Wood R J 2013 Science 340, 603-607.

Maeda M \& Liu H 2013 J. Biomech. Sci. Eng. 8, 344-355.

Mayo D B \& Leishman J G 2010 J. Am. Helicopter Soc. 55, 025001(5pp).

Minami K, Suzuki K \& Inamuro T 2015 Fluid Dyn. Res. 47, 015505 (17pp).

Mittal R \& Iaccarino G 2005 Annu. Rev. Fluid Mech. 37, 239-261.

Noda R, Nakata T \& Liu H 2014 Acta Mech. Sin. 30, 819-827.

Ota K, Suzuki K \& Inamuro T 2012 Fluid Dyn. Res. 44, 045504 (27pp).

Ozen C A \& Rockwell D 2012 Exp. Fluids 52, 207-223.

Pesavento U \& Wang Z J 2009 Phys. Rev. Lett. 103, 118102 (4pp).

Peskin C S 1972 J. Comput. Phys. 10, 252-271.

Peskin C S 2002 Acta Numerica 11, 479-517.

Ristroph L, Bergou A J, Guckenheimer J, Wang Z J \& Cohen I 2011 Phys. Rev. Lett. 106, 178103 (4pp).

Ristroph L \& Childress S 2014 J. R. Soc. Interface 11, 20130992 (7pp).

Succi S 2001 The Lattice Boltzmann Equation for Fluid Dynamics and Beyond (Oxford: Oxford University Press).

Sui Y, Chew Y T, Roy P \& Low H T 2008 J. Comput. Phys. 227, 6351-6371.

Suzuki K \& Inamuro T 2011 Comput. Fluids 49, 173-187.

Suzuki K, Minami K \& Inamuro T 2015 J. Fluid Mech. 767, 659-695.

Usherwood J R \& Ellington C P $2002 a$ J. Exp. Biol. 205, 1547-1564.

Usherwood J R \& Ellington C P 2002b J. Exp. Biol. 205, 1565-1576.

Wang Z, Fan J \& Luo K 2008 Int. J. Multiphase flow 34, 283-302.

Wu J \& Shu C 2009 J. Comput. Phys. 228, 1963-1979.

Wu J, Yang S C, Shu C, Zhao N \& Yan W W 2015 J. Fluids Struct. 54, 247-262.

Yokoyama N, Senda K, Iima M \& Hirai N 2013 Phys. Fluids 25, 021902 (24pp).

Zhang X, Lua K B, Chang R, Lim T T \& Yeo K S 2014 Int. J. Mod. Phys. Conf. Ser. 34, 1460384 (12pp).

Zheng L, Hedrick T \& Mittal R 2013 Bioinsp. Biomim. 8, 036001 (13pp). 